\title{
The Extreme Spin of the Black Hole in Cygnus X-1
}

\section{Citation}

Gou, Lijun, Jeffrey E. McClintock, Mark J. Reid, Jerome A. Orosz, James F. Steiner, Ramesh Narayan, Jingen Xiang, Ronald A. Remillard, Keith A. Arnaud, and Shane W. Davis. 2011. "The Extreme Spin of the Black Hole in Cygnus X-1." The Astrophysical Journal 742, no. 2: 85.

\section{Published Version}

doi:10.1088/0004-637X/742/2/85

\section{Permanent link}

http://nrs.harvard.edu/urn-3:HUL.InstRepos:13041331

\section{Terms of Use}

This article was downloaded from Harvard University's DASH repository, and is made available under the terms and conditions applicable to Other Posted Material, as set forth at http:// nrs.harvard.edu/urn-3:HUL.InstRepos:dash.current.terms-of-use\#LAA

\section{Share Your Story}

The Harvard community has made this article openly available.

Please share how this access benefits you. Submit a story.

\section{Accessibility}




\title{
THE EXTREME SPIN OF THE BLACK HOLE IN CYGNUS X-1
}

\author{
Lijun Gou ${ }^{1}$, Jeffrey E. McClintock ${ }^{1}$, Mark J. Reid ${ }^{1}$, Jerome A. Orosz ${ }^{2}$, James F. Steiner ${ }^{1}$, \\ Ramesh Narayan $^{1}$, Jingen Xiang ${ }^{1}$, Ronald A. Remillard ${ }^{3}$, Keith A. Arnaud ${ }^{4,5}$, and Shane W. Davis 6 \\ ${ }^{1}$ Harvard-Smithsonian Center for Astrophysics, Cambridge, MA 02138, USA \\ ${ }^{2}$ Department of Astronomy, San Diego State University, San Diego, CA 92182, USA \\ ${ }^{3}$ Kavli Institute for Astrophysics and Space Research, MIT, Cambridge, MA 02139, USA \\ ${ }^{4}$ CRESST, NASA Goddard Space Flight Center, Greenbelt, MD 20771, USA \\ ${ }^{5}$ Astronomy Department, University of Maryland, College Park, MD 20742, USA \\ ${ }^{6}$ Canadian Institute for Theoretical Astrophysics, University of Toronto, Toronto, ON M5S 3H8, Canada \\ Received 2011 June 17; accepted 2011 September 12; published 2011 November 9
}

\begin{abstract}
The compact primary in the X-ray binary Cygnus X-1 was the first black hole to be established via dynamical observations. We have recently determined accurate values for its mass and distance, and for the orbital inclination angle of the binary. Building on these results, which are based on our favored (asynchronous) dynamical model, we have measured the radius of the inner edge of the black hole's accretion disk by fitting its thermal continuum spectrum to a fully relativistic model of a thin accretion disk. Assuming that the spin axis of the black hole is aligned with the orbital angular momentum vector, we have determined that Cygnus X-1 contains a near-extreme Kerr black hole with a spin parameter $a_{*}>0.95(3 \sigma)$. For a less probable (synchronous) dynamical model, we find $a_{*}>0.92(3 \sigma)$. In our analysis, we include the uncertainties in black hole mass, orbital inclination angle, and distance, and we also include the uncertainty in the calibration of the absolute flux via the Crab. These four sources of uncertainty totally dominate the error budget. The uncertainties introduced by the thin-disk model we employ are particularly small in this case given the extreme spin of the black hole and the disk's low luminosity.
\end{abstract}

Key words: accretion, accretion disks - black hole physics - stars: individual (Cygnus X-1) - X-rays: binaries

Online-only material: color figures

\section{INTRODUCTION}

In our two companion papers (Reid et al. 2011; Orosz et al. 2011), we report accurate measurements of the distance, black hole mass, and orbital inclination angle for the black hole binary system Cygnus X-1. Herein, we use these results to determine the spin of the black hole primary by fitting the thermal component of its X-ray spectrum to our implementation of the Novikov-Thorne $\operatorname{model}^{7}$ of a thin accretion disk (Li et al. 2005).

Cygnus X-1 was discovered at the dawn of X-ray astronomy (Bowyer et al. 1965) and is one of the brightest and most persistent celestial X-ray sources. Its compact primary was the first object to be established as a black hole via dynamical observations (Webster \& Murdin 1972; Bolton 1972). For several decades, the source has been extensively observed at radio, optical, ultraviolet, and X-ray wavelengths.

Cygnus X-1 is typically in a hard spectral state, but occasionally it switches to a soft state, which may persist for up to a year (see Figure 1). It is only in this soft state, when the disk spectrum is prominent, that one can measure the spin using the continuum-fitting method we employ. In this soft state, which corresponds to the steep power-law (SPL) state, ${ }^{8}$ a

\footnotetext{
7 Note that we have corrected the original Novikov-Thorne equations (Novikov \& Thorne 1973) for the problem identified by Riffert \& Herold (1995). The term "Novikov-Thorne" here refers to a relativistic and geometrically thin accretion disk in Kerr geometry with a no-torque boundary condition at the disk's inner edge.

8 Throughout, we use the X-ray states defined by Remillard \& McClintock (2006): hard, thermal dominant (TD), steep power-law (SPL), and intermediate states. In the alternative state classification scheme of Homan \& Belloni (2005), the only states reached by Cygnus X-1 are the low/hard, hard-intermediate, and soft-intermediate, which correspond, respectively, to our hard, intermediate (i.e., hard:SPL), and SPL states.
}

strong Compton component is always present. Although Cygnus $\mathrm{X}-1$ has been observed on thousands of occasions, it has never been observed to reach the thermal dominant (TD) state, the state that is most favorable for the measurement of spin via the continuum-fitting method (Steiner et al. 2009a).

Following the pioneering work of Zhang et al. (1997), we measure black hole spin by estimating the inner radius of the accretion disk $R_{\text {in }}$. In this approach to measuring spin, one identifies $R_{\text {in }}$ with the radius of the innermost stable circular orbit $R_{\mathrm{ISCO}}$, which is predicted by general relativity. $R_{\mathrm{ISCO}}$ is a monotonic function of the dimensionless spin parameter $a_{*}$, decreasing from $6 \mathrm{GM} / \mathrm{c}^{2}$ to $1 \mathrm{GM} / \mathrm{c}^{2}$ as spin increases from $a_{*}=0$ to $a_{*}=1$ (Bardeen et al. 1972). ${ }^{9}$ This relationship between $a_{*}$ and $R_{\mathrm{ISCO}}$ is the foundation for measuring spin by either the continuum-fitting method (Zhang et al. 1997) or by the Fe K $\alpha$ method (Fabian et al. 1989; Reynolds \& Nowak 2003).

The identification of $R_{\text {in }}$ with $R_{\mathrm{ISCO}}$ is strongly supported by the abundant empirical evidence that the inner radius of the disk in the soft state of a black hole binary does not appear to change even as the temperature and luminosity change. LMC X-3 is a prime example; its inner-disk radius has been shown to be stable to several percent over a period of 26 years (Done et al. 2007; Steiner et al. 2010). Strong theoretical support for identifying $R_{\text {in }}$ with $R_{\mathrm{ISCO}}$ is provided by magnetohydrodynamic simulations of thin accretion disks, which show the disk emission falling off rapidly inside the innermost stable circular orbit (ISCO) (Shafee et al. 2008; Reynolds \& Fabian 2008; Penna et al. 2010; Kulkarni et al. 2011; Noble et al. 2010).

In our early work on measuring spin, we relied solely on TD-state data (e.g., Shafee et al. 2006; McClintock et al. 2006;

\footnotetext{
$9 \quad a_{*} \equiv c J / G M^{2}$ with $\left|a_{*}\right| \leqslant 1$, where $M$ and $J$ are respectively the black hole mass and angular momentum.
} 
Table 1

Journal of the Observations That Yielded Spectra SP1, SP2, and SP3 ${ }^{\mathrm{a}}$

\begin{tabular}{|c|c|c|c|c|c|c|c|c|}
\hline No. & Mission & Detector & $\begin{array}{c}E_{1}-E_{2} \\
(\mathrm{keV})\end{array}$ & UT & $\begin{array}{l}T_{\exp } \\
\text { (s) }\end{array}$ & $\begin{array}{c}I \\
(\mathrm{Crab})\end{array}$ & $\mathrm{SH}$ & $\phi$ \\
\hline SP1 & $A S C A$ and $R X T E$ & GIS and PCA & $0.7-8.0$ and $2.5-45.0$ & 1996 May 30 06:43:16 and 07:51:29 & 2547 and 2240 & 0.80 & 0.64 & 0.74 \\
\hline SP2 & Chandra and $R X T E$ & ACIS(TE)/HETG and PCA & $0.5-10.0$ and $2.5-45.0$ & 2010 July 22 16:21:22 and 17:04:01 & 2146 and 3808 & 1.31 & 0.54 & 0.24 \\
\hline SP3 & Chandra and $R X T E$ & ACIS(CC)/HETG and PCA & $0.5-10.0$ and $2.5-45.0$ & 2010 July 24 17:21:43 and 17:43:00 & 900 and 3904 & 1.01 & 0.61 & 0.61 \\
\hline
\end{tabular}

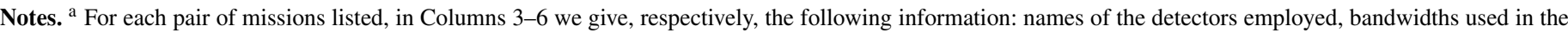

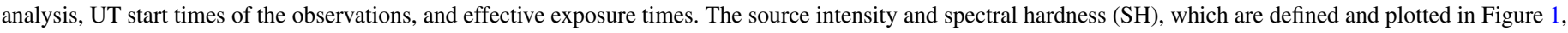

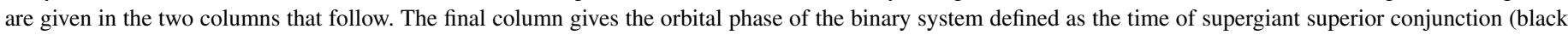
hole beyond O-star), which occurred on heliocentric Julian Day 2441874.71 (Brocksopp et al. 1999).

Liu et al. 2008). More recently, using our empirical model of Comptonization SIMPL (Steiner et al. 2009b), we have shown that one can obtain values of the inner disk radius using SPL data that are consistent (within $\sim 5 \%$ ) with those obtained using TD data if the scattering fraction ${ }^{10} f_{\text {SC }} \lesssim 25 \%$ (Steiner et al. 2009a). Consequently, we are now able to routinely and reliably obtain spin estimates for sources in the SPL state (Gou et al. 2009; Steiner et al. 2011) if the scattering fraction is not too extreme. This development has paved the way for measuring the spin of Cygnus X-1, which has never been observed in the TD state.

For the continuum-fitting method to succeed, it is essential to have an accurate value for the black hole mass $M$ in order to express the radius appropriately as the dimensionless quantity $R_{\mathrm{ISCO}} c^{2} / G M$. For the method to succeed, it is furthermore essential to know the disk luminosity, and therefore to have accurate values for the distance $D$ and the disk inclination angle $i$, which we infer by assuming that the orbital angular momentum vector and black hole spin axis are aligned (see Section 7.4). We measured these three critical parameters in a two-step process. First, using the Very Long Baseline Array (VLBA), we determined a model-independent distance via trigonometric parallax that is accurate to $6 \%: D=1.86_{-0.11}^{+0.12} \mathrm{kpc}$ (Reid et al. 2011). Second, using this accurate distance estimate to constrain the radius of the companion star, we then modeled an extensive collection of optical data. For our favored asynchronous model, Model $\mathcal{D}$, we find $M=14.8 \pm 1.0 M_{\odot}$ and $i=27.1 \pm 0.8$ (Orosz et al. 2011). With the values of these three input parameters in hand, and using three soft-state X-ray spectra, we determined the spin of Cygnus X-1; our final value is presented in Section 6.

Spin measurements have previously been obtained using the continuum-fitting method for eight black holes (McClintock et al. 2011). Three have low to moderate spins, $a_{*} \lesssim 0.5$; four have high spins, $a_{*} \sim 0.7-0.9$; and one of them, the archetypal microquasar GRS 1915+105 (Mirabel \& Rodríguez 1994; Fender \& Belloni 2004), is a near-extreme Kerr hole with $a_{*}>0.98$ (McClintock et al. 2006; Blum et al. 2009).

The paper is organized as follows. In Section 2, we discuss our observations and the selection and reduction of the X-ray spectral data. The analysis of these data and our results are presented, respectively, in Sections 3 and 4 for our adopted model, and the robustness of these results is discussed in Section 5. (Additional analysis work and results for several preliminary models are presented in the appendices.) In Section 6, we first determine the error in the spin parameter due to the combined

\footnotetext{
${ }^{10} f_{\mathrm{SC}}$ is the fraction of the thermal seed photons that are scattered into the power-law tail, and it is the normalization parameter of our XSPEC model SIMPL/SIMPLR (see Section 3.2).
}

uncertainties in $D, M, i$, and the absolute flux calibration, and we then present our final value of $a_{*}$ and confidence limits. Seven distinct topics are addressed in Section 7, and in Section 8 we offer our conclusions.

\section{DATA SELECTION, OBSERVATIONS, AND DATA REDUCTION}

Very few spectra of Cygnus X-1 exist that are suitable for the measurement of the black hole's spin for reasons we now discuss. A typical soft-state (and SPL) spectrum is comprised of three principal elements: a thermal component, a power-law component, and a reflected component, which includes the Fe $\mathrm{K} \alpha$ emission line. Three such spectra are analyzed in detail and illustrated in Section 3. It is apparent from an inspection of these spectra that the spectral coverage must extend to at least $30 \mathrm{keV}$ in order to constrain the strong power-law and reflection components. At the same time, because the temperature of the thermal component is consistently low $(k T \sim 0.5 \mathrm{keV}$; Appendix A), one also requires coverage down to $\approx 1 \mathrm{keV}$ in order to constrain this crucial thermal component, which is partially absorbed at low energies by intervening gas. The rarity of spectra that meet these requirements became clear to us following our exhaustive search of the thousands of spectra of Cygnus X-1 that are contained in the HEASARC data archive. To our surprise, we found only a single spectrum, SP1 (Table 1), that is suitable for the measurement of black hole spin. ${ }^{11}$ It was obtained on 1996 May 30 in an observation made simultaneously using the Advanced Satellite for Cosmology and Astrophysics (ASCA) and the Rossi X-ray Timing Explorer (RXTE).

There is a second, important reason for the paucity of suitable spectra, namely, that Cygnus X-1 is seldom in the required disk-dominated state. This fact is illustrated in Figure 1, which summarizes the behavior of Cygnus X-1 since 1996 as observed using the RXTE all-sky monitor (ASM). As indicated in the figure, we select only those data for which the spectral hardness $\mathrm{SH}<0.7$, which occurs $<10 \%$ of the time. Fortunately, Cygnus X-1 entered its soft state in mid-2010, and we obtained two additional broadband spectra on July 22 and 2010 July 24 by making simultaneous observations using the Chandra $X$-ray Observatory and $R X T E$ (Table 1). The times of these two observations and the $A S C A / R X T E$ observation are indicated by arrows in Figure 1, and the corresponding ASM measures of

\footnotetext{
11 We decided not to use the Swift X-Ray Telescope (XRT)/Burst Alert Telescope spectra, which provide broadband coverage, because (1) the XRT timing spectra (e.g., Swift ID 00031651005) show strong residual features in the energy range 1-3 keV; (2) the low-rate photodiode spectrum (Swift ID 00101469000) is poorly calibrated (Romano et al. 2005); and (3) Swift provides no coverage at all from 10 to $15 \mathrm{keV}$.
} 


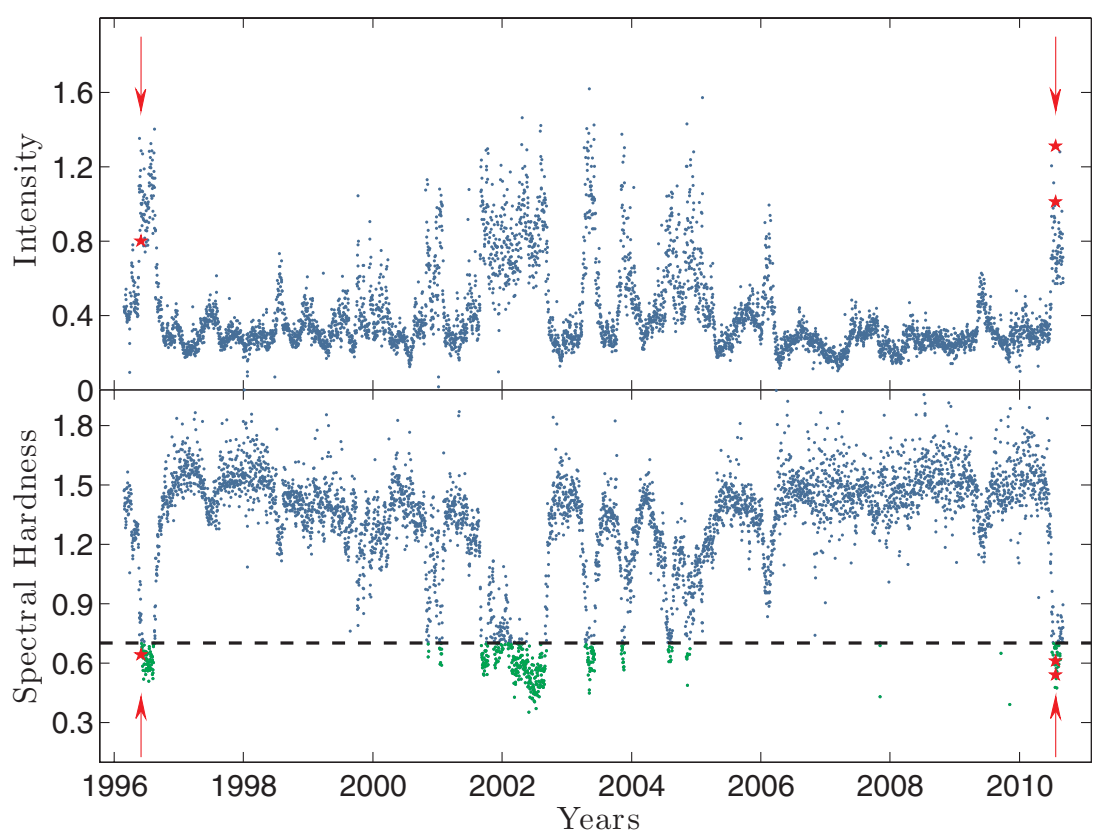

Figure 1. Fourteen-year record for Cygnus X-1 of X-ray intensity relative to the Crab nebula (top) and spectral hardness SH (bottom). The hardness is defined as the ratio of counts detected in a hard X-ray band (5-12 keV) to those detected in a soft (1.5-5 keV) band. We consider data suitable for the measurement of spin only when the spectral hardness is below the dashed line $(\mathrm{SH}<0.7)$, which is an empirical choice. Shown plotted as red stars are the intensity and hardness of the source for each of the three selected observations, SP1 in 1996, and SP2 and SP3 in 2010. While a useful diagnostic for the purposes of data selection, the RXTE/ASM sky survey data shown here are unsuitable for the measurement of spin.

(A color version of this figure is available in the online journal.)

intensity and spectral hardness are plotted as red stars. Detailed information on these three observations is summarized in Table 1. Three spectra were derived from these observations: the 1996 archival ASCA/RXTE spectrum SP1, and the two Chandra/RXTE spectra, SP2 and SP3, which were obtained in 2010.

We first focus on spectrum SP1, which has been described in detail by others (Dotani et al. 1997; Cui et al. 1998; Gierliński et al. 1999). We include in our analysis all of the data collected by both ASCA and RXTE even though the observations were strictly simultaneous for only about $1000 \mathrm{~s}$. This is a reasonable approach because both missions show that the source intensity was stable during the entire observing period (see Figure 4 in Gierliński et al. 1999). For ASCA, we consider only data collected by the second Gas Imaging Spectrometer (GIS) detector, GIS2 (the GIS3 data were excluded because of an unexplained residual feature in the spectrum near $1 \mathrm{keV}$ ). We disregard the Solid-state Imaging Spectrometer data because of data-rate limitations. For RXTE, we use all of the data collected by all five Proportional Counter Array (PCA) detectors. For the main analysis, we disregard the RXTE High Energy $\mathrm{X}$-ray Timing Experiment (HEXTE) data because the useful bandwidth of the PCA, which extends to $45 \mathrm{keV}$, already provides more than adequate energy coverage. We demonstrate this fact in Section 5.2 where we show that the inclusion of HEXTE data has no significant affect on our results.

The GIS spectra were extracted following the standard procedures described in The ASCA Data Reduction Guide. ${ }^{12}$ The GIS2 spectrum was fitted over the energy range $0.7-8.0 \mathrm{keV}$. We did not correct the effective area of the detector because the instrument team had already done this calibration to an accuracy

12 The manual is available at

http://heasarc.gsfc.nasa.gov/docs/asca/abc/abc.html. of 3\% using the standard Crab spectrum (Toor \& Seward 1974; Makishima et al. 1996; Steiner et al. 2010).

We reduced the RXTE PCA data following the same procedures described in McClintock et al. (2006). Data reduction was performed with standard tools from the HEASOFT package provided by NASA. The critical steps in determining the PCA background and making the response files for spectral analysis utilized HEASOFT version 6.10. X-ray spectra were extracted from the Standard 2 telemetry mode, which provides coverage of the full PCA bandpass every $16 \mathrm{~s}$. Data from all Xe gas layers of PCU-2 were combined to make each spectrum. The background spectrum was determined with the "bright source" model. Redistribution matrix files and ancillary response files were freshly generated individually for each PCU layer and then combined into a single response file using the tool pcarsp. We used the PCA response matrices ${ }^{13}$ v11.7 released on $2009 \mathrm{Au}-$ gust 17 , which allowed us to obtain reliable fits over the energy range $2.55-45.0 \mathrm{keV}$.

We corrected for the effective area of the PCA using the spectrum of the Crab Nebula as a standard source and using our recently adopted and improved method, which is described in Steiner et al. (2010). Specifically, we compared the Crab spectrum of Toor \& Seward (1974) $(\Gamma=2.1$ and $N=$ 9.7 photons $\mathrm{s}^{-1} \mathrm{keV}^{-1}$ ) to parameters obtained by analyzing proximate archival observations of the Crab. In this way, we determined a pair of correction factors for spectrum SP1: a normalization correction factor $C_{\mathrm{TS}}=1.123$ (the ratio of the observed normalization to that of Toor \& Seward) and a correction to the slope of the power law, $\Delta \Gamma_{\mathrm{TS}}=0.023$ (the difference between the observed value of $\Gamma$ and that of Toor \& Seward). These corrections were applied in all of our analysis work via a custom XSPEC multiplicative model CRABCOR.

\footnotetext{
${ }^{13}$ For a description of the latest response files, see http://www.universe.nasa.gov/xrays/programs/rxte/pca/doc/rmf/pcarmf-11.7.
} 
We also corrected the detector count rates for dead time by the factor 1.048 .

We turn now to the recent Chandra/RXTE observations (Table 1; spectra SP2 and SP3). The observations performed by the two spacecraft were mostly simultaneous, overlapping for about $4 \mathrm{ks}$, while the total duration of each Chandra observation was $\approx 6 \mathrm{ks}$. Because the source intensity for both observations was constant to within $10 \%$, we included in our analysis all of the Chandra data, plus the strictly simultaneous $R X T E$ PCA data.

Our Chandra observations were made using the High-Energy Transmission Grating (HETG) and the Advanced Camera for Imaging and Spectroscopy (ACIS; Canizares et al. 2005; Garmire et al. 2003). The data-rate limitation of the detectors makes it challenging to observe a bright and variable source like Cygnus X-1. The principal problem is "pile-up," i.e., the registering of two or more photons in the same or adjacent pixels within a single frame time. Given the uncertainties, and as a hedge, we performed the pair of Chandra observations using two different instrumental configurations, Timed Exposure (TE) mode and Continuous Clocking (CC) faint mode, which have contrasting virtues and limitations.

In reducing the Chandra TE-mode data, we followed the method described by Smith et al. (2002). In order to avoid saturating the telemetry, only the data for the High Energy Grating (HEG; -1 order) and Medium Energy Grating (MEG; +1 order) components of the HETG were recorded. The data for the readout streak on the same side of the HEG and MEG spectra were also recorded. We extracted the "streak" and background spectra following the recommended procedures. ${ }^{14} \mathrm{Al}$ though the net exposure time for the HETG spectrum is $2.15 \mathrm{ks}$, the effective exposure time for the streak spectrum is only about $8 \mathrm{~s} .{ }^{15}$ For this spectrum, we estimate that less than $3 \%$ of the events are affected by pileup, and we therefore use the full $0.5-10 \mathrm{keV}$ bandwidth. For the dispersed grating spectrum, we only included data for which $<5 \%$ of the events are piled up; for the HEG and MEG, respectively, these data are in the energy ranges $0.7-0.9 \mathrm{keV}$ and $7.0-10.0 \mathrm{keV}$.

In the CC mode, the frame time is reduced to about $3 \mathrm{~ms}$ (compared to $1.3 \mathrm{~s}$ for the TE mode) by continuously transferring the data from the image array to the frame-store array. While this essentially eliminates pileup, the details of the spatial distribution in the columns are then lost due to the collapse of the two-dimensional image into a one-dimensional image. Again, only the orders of the HEG and MEG spectra mentioned above were recorded. The spectra were extracted following the standard procedures. ${ }^{16}$ We fitted these data over the full energy range $0.5-10.0 \mathrm{keV}$, except for the 1.3-2.0 keV chip gap in the MEG spectrum. A downside of the $\mathrm{CC}$ mode is that its calibration is less certain than that of the TE mode.

For RXTE, lacking data from all five PCUs, we elected to use only the data from what historically has been the bestcalibrated detector, PCU2. (One obtains essentially the same results for the Chandra/RXTE spectra using any combination of the available PCUs; likewise, for the ASCA/RXTE spectrum, it is unimportant whether one uses all PCUs, as we did, or PCU2 alone.) As described above, we corrected the effective area of the detector. For both observations, the normalization correction is $C_{\mathrm{TS}}=1.073$, and the power-law slope correction

\footnotetext{
14 http://cxc.harvard.edu/ciao/threads/streakextract/

15 The procedure for calculating this exposure time can be found at http://cxc.harvard.edu/ciao/threads/streakextract/index.html\#exposure.

$16 \mathrm{http}: / / \mathrm{cxc}$. harvard.edu/ciao/threads/spectra_hetgacis/
}

is $\Delta \Gamma_{\mathrm{TS}}=0.029$. The dead time corrections for SP2 and SP3 are, respectively, 1.052 and 1.044 .

Finally, we included systematic errors in the count rates in each pulse height analyzer channel to account for uncertainties in the instrumental responses: $1 \%$ for GIS2 and $0.5 \%$ for all the PCUs. All the Chandra data were binned to achieve a minimum number of counts per channel of 200; no systematic error was included because the statistical error is large.

\section{DATA ANALYSIS}

A typical spectrum of Cygnus X-1 is comprised of three principal elements: a thermal component, a power-law component, and a reflected component that includes the $\mathrm{Fe} \mathrm{K} \alpha$ emission line. The structures in the X-ray source that give rise to these components, namely, the accretion disk and its corona, are illustrated in Figure 2. The spectral components themselves, in relation to the total observed spectrum, are shown plotted in Figure 3, which illustrates the results of the relativistic analysis described in this section.

The data analysis and model fitting throughout this paper were performed using $\mathrm{XSPEC}^{17}$ version 12.6.0 (Arnaud 1996) and, unless otherwise indicated, errors are everywhere reported at the $1 \sigma$ level of confidence. In this section (and throughout Appendices A and B), we fix the key input parameters $D, M$, and $i$ at their fiducial values, which are given in Section 1 .

\subsection{Seven Preliminary Models}

Our adopted model that is featured below, and upon which all of our results are based, was constructed by working in detail through a progression of seven preliminary models. We now briefly comment on these models, which are presented in full in Appendices A and B.

Nonrelativistic models. The central component of our three nonrelativistic models, Models NR1-NR3, is the familiar accretion-disk model component DISKBB ${ }^{18}$ (Mitsuda et al. 1984; Makishima et al. 1986), which does not include any relativistic effects or the effects of spectral hardening, and which has an inappropriate boundary condition at the disk's inner edge (Zimmerman et al. 2005). We nevertheless employed DISKBB as an exploratory tool because it has been widely used for decades, and it therefore allows us to compare our reduction/analysis results (for a spectrum of interest) to published results. Furthermore, this familiar model returns a direct and useful estimate of the temperature at the inner edge of the disk, which we use in order to make comparisons between Cygnus X-1 and other black hole binaries. The details of this nonrelativistic analysis and the satisfying results obtained for Model NR3 - namely, consistent values of the inner-disk radius and temperature for our three spectra-are presented in Appendix A.

Relativistic models. Similarly, in addition to our adopted relativistic model (Section 3.2), in Appendix B we present our analysis and results for four relativistic models, Models R1-R4, that are built around our fully relativistic accretion-disk model component KERRBB2, which we describe below. This component is a direct replacement for DISKBB, returning two fit parameters, namely, the spin and the mass accretion rate (instead of the temperature and radius of the inner disk). The four models

\footnotetext{
$17 \mathrm{XSPEC}$ is available at http://heasarc.gsfc.nasa.gov/xanadu/xspec/

18 For descriptions of the XSPEC models, see

http://heasarc.gsfc.nasa.gov/docs/xanadu/Xspec/manual/XspecModels.html.
} 


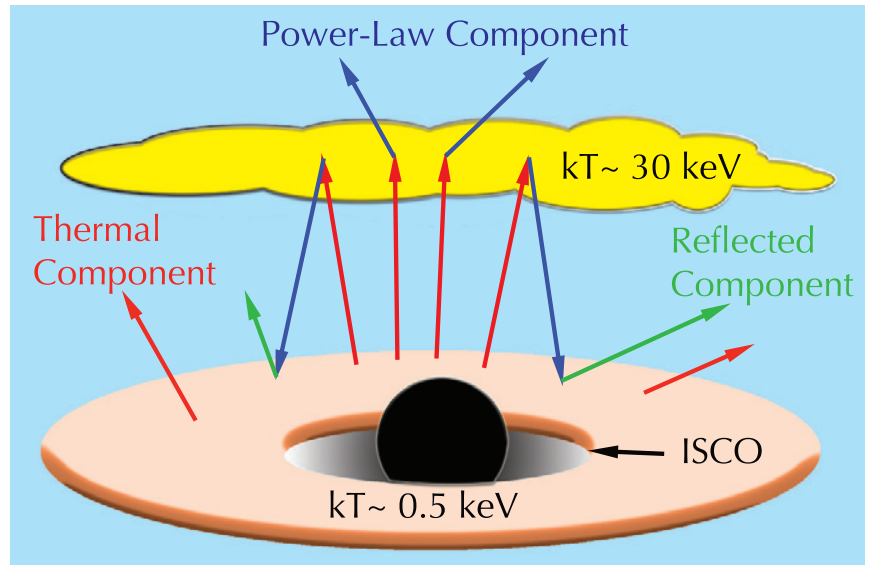

Figure 2. Schematic sketch of the X-ray source (adapted from a sketch provided by R. Reis). The accretion disk (pink) is truncated at the ISCO, leaving a dark gap between the disk's inner edge and the black hole's event horizon (black). Shown hovering above the optically thick disk is its tenuous scattering corona (yellow). As indicated by the arrows, the disk supplies the thermal component of emission, which is Compton scattered into a power-law component by hot electrons in the corona. Approximately half of this latter component illuminates the disk, thereby generating the reflected component.

progress sequentially in the sense that Model R1 is the most primitive and Model R4 is the most advanced. This sequence builds toward our adopted model. We have chosen to present our results for these preliminary relativistic models, in addition to those for our adopted model, because doing so demonstrates that our modeling of the critical thermal component, and the extreme spin it delivers for Cygnus X-1, are insensitive to the details of the analysis.

\subsection{Our Adopted Model}

The model we employ is a culmination of Models R1-R4 in the sense that it is the most advanced and physically realistic model. The schematic sketch of the X-ray source in Figure 2 illustrates the various model components and their interplay.
The structure of our adopted model, naming all the components that comprise it, is expressed as follows:

\section{$\mathrm{CRABCOR} * \mathrm{CONST} * \mathrm{TBABS}[\mathrm{SIMPLR} \otimes \mathrm{KERRBB} 2$ $+\mathrm{KERRDISK}+\mathrm{KERRCONV} \otimes($ IREFLECT $\otimes$ SIMPLC)].}

As described in detail below, SIMPLR generates the powerlaw component using the seed photons supplied by the single thermal component KERRBB2, while the reflection component is likewise generated in turn by IREFLECT acting solely on the power-law component (i.e., IREFLECT does not act on the thermal component). Furthermore, the model fits for a single value of $a_{*}$, which appears as the key fit parameter in three model components: KERRBB2, KERRDISK, and KERRCONV.

We now discuss in turn the model's three principal components - thermal, power law, and reflected-and their interrelationships.

Thermal component. the centerpiece of our adopted model is our accretion-disk model KERRBB2, which includes all relativistic effects, self-irradiation of the disk ("returning radiation"), and limb darkening (Li et al. 2005). The effects of spectral hardening are incorporated into the basic model KERRBB via a pair of look-up tables for the hardening factor $f$ corresponding to two representative values of the viscosity parameter: $\alpha=0.01$ and 0.1 (McClintock et al. 2006). Motivated by observational data obtained for dwarf novae (Smak 1998, 1999) and soft X-ray transients (Dubus et al. 2001), and the results of global general relativistic magnetohydrodynamic (GRMHD) simulations (Penna et al. 2010), throughout this work we adopt $\alpha=0.1$ as our fiducial value; meanwhile, in Section 5.4 we examine the effects on our results of using $\alpha=0.01$ in place of $\alpha=0.1$. The entries in the look-up tables for $f$ were computed using both KERRBB and a second relativistic disk model BHSPEC (Davis et al. 2005; Davis \& Hubeny 2006). We refer to the model KERRBB plus this table, and the subroutine that reads it, as KERRBB2 (McClintock et al. 2006). As noted above, the model KERRBB2 has just two fit parameters, namely, the black hole spin parameter $a_{*}$ and the mass accretion rate $M$ (or equivalently, $a_{*}$ and the

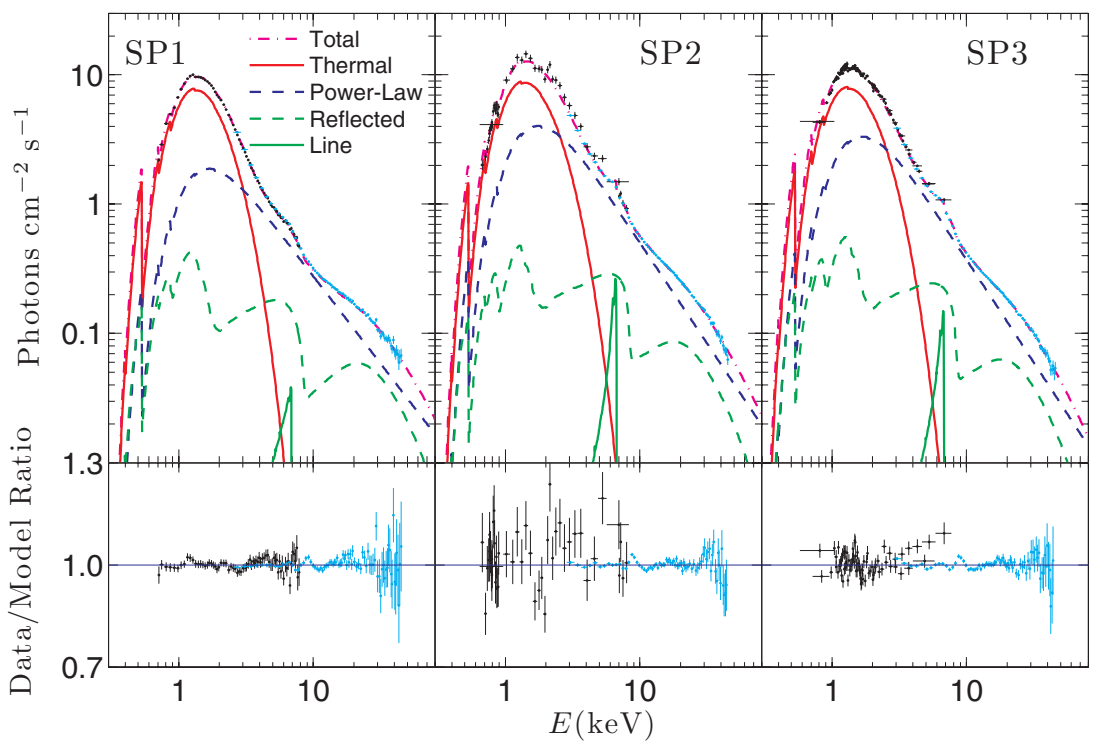

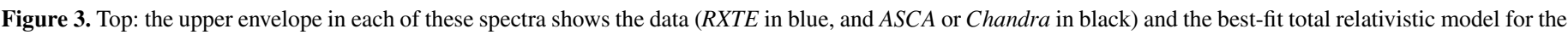

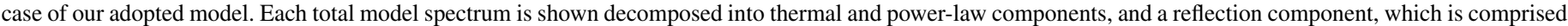

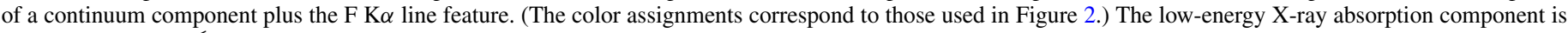

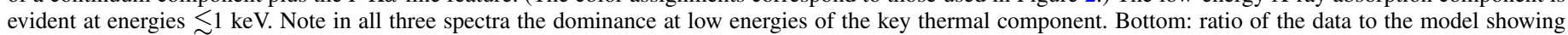
deviations between the two. 
Eddington-scaled bolometric luminosity, $\left.l \equiv L_{\text {bol }}\left(a_{*}, \dot{M}\right) / L_{\text {Edd }}\right)$. For the calculations reported in this paper, we included the effects of limb darkening and returning radiation. We set the torque at the inner boundary of the accretion disk to zero (as appropriate when $D, M$, and $i$ are held fixed), allowed the mass accretion rate to vary freely, and fitted directly for the spin parameter $a_{*}$.

Power-law component. The first term in the sum, SIMPLR $\otimes$ KERRBB2, models the power-law component and the observed thermal component in combination. This dominant part of the spectrum (see Figure 3 ) is computed by convolving KERRBB2, which describes the seed photon distribution (i.e., the thermal component prior to being scattered), with SIMPLR. The convolution model SIMPLR is a slightly modified version of SIMPL (Steiner et al. 2009b) that is appropriate when including a separate and additive reflection component (Steiner et al. 2011). The parameters of SIMPLR (and SIMPL) are the power-law photon index $\Gamma$ and the scattered fraction, $f_{\mathrm{SC}}$, which is the fraction of the seed photons that are scattered into the power-law tail. As used here, SIMPLR describes a corona that sends approximately half the scattered seed photons outward toward the observer and the remainder downward toward the disk, thereby generating the reflected component (see below). Thus, we assume that the power-law component illuminating the disk is the same as the component we observe.

Reflected component. The second and third terms in the sum model the reprocessed emission from the disk that results from its illumination by the power-law component. The model for the illuminating power-law component itself (the term on the far right) is SIMPLC, which is equivalent to SIMPLR $\otimes$ KERRBB2 minus the unscattered thermal component (Steiner et al. 2011). This power-law component is acted on by IREFLECT, which is a convolution reflection model with the same properties as its widely used parent, the additive reflection model PEXRIV (Magdziarz \& Zdziarski 1995). Concerning IREFLECT, we (1) free the reflection scaling factor ${ }^{19} s$ while restricting its range to negative values, thereby computing only the reflected spectrum, while assuming that the corona is a thin slab that hugs the disk and emits isotropically; (2) set the elemental-abundance switch to unity, which corresponds to solar abundances, while allowing the iron abundance to be free; (3) link the photon index to the value returned by SIMPLR; and (4) fix the disk temperature at $6.0 \times 10^{6} \mathrm{~K}$, the temperature $T_{\text {in }}$ returned by DISKBB (see Appendix A). The model IREFLECT $\otimes$ SIMPLC returns a reflected spectrum that contains sharp absorption features and no emission lines. To complete the model of the reflected component, we follow Brenneman \& Reynolds (2006) and employ the line model KERRDISK and the convolution smearing model KERRCONV, both of which treat $a_{*}$ as a free fit parameter. ${ }^{20}$ These models allow the emissivity indices to differ in the inner and outer regions of the disk. For simplicity, and because this parameter is unknown with values that vary widely from application to application, we use an unbroken emissivity profile with a single index $q$. We tie together all the

\footnotetext{
19 The reflection scaling factor $s$ in IREFLECT (and PEXRIV) is linked to the reflection constant parameter $x$ in SIMPLR via the relation $x=1+|s|$ (where $|s|$ is the absolute value of $s$ ). In the limiting case of $x=2$, half of the scattered photons are redirected downward and illuminate the disk. In the limiting case of $x=1$, none of the Compton-scattered photons strike the disk (Steiner et al. 2011). For the preliminary models described in the appendices that use the additive model PEXRIV, the scaling factor $s$ is fixed at -1 .

20 We performed tests with a newer version of these models, RELLINE and RELCONV (Dauser et al. 2010), which are interpolated on a finer grid, and found that our results presented in Section 4 (and elsewhere) are essentially unchanged.
}

common parameters of KERRDISK and KERRCONV, including the two principal parameters, namely, $a_{*}$ and $q$.

The three multiplicative model components are, respectively, (1) CRABCOR, which corrects for detector effects (see Section 2); (2) CONST, which reconciles the calibration differences between the detectors (throughout the paper, we fix the normalization of the RXTE/PCU2 detector and float the normalization of the ASCA GIS and Chandra HETG/ACIS detectors); and (3) TBABS, ${ }^{21}$ which models low-energy absorption (Wilms et al. 2000). Concerning TBABS, throughout the paper we allow $N_{\mathrm{H}}$ to vary because the column density is well determined by the data and $N_{\mathrm{H}}$ is known to vary by several percent for all three wellstudied supergiant black hole binaries, namely, Cygnus X-1 (Hanke et al. 2009; also see Section 4), M33 X-7 (Liu et al. 2008), and LMC X-1 (Hanke et al. 2010).

\section{RESULTS}

The fit results for our adopted model are given in Table 2, and the three fitted spectra together with their spectral components are shown in Figure 3. The fits are all good, with $\chi_{v}^{2}$ ranging from 1.17 to 1.28 , and the results in Table 2 are in good agreement with those obtained using Models R1-R4 (see Appendix B and Tables 9-12). As in the case of Models R1-R4, the spin parameter is high with $a_{*}>0.99$ for all three spectra. This is the principal result of this section. In the following section, we examine the robustness of this result, and in Section 6 we fold in the uncertainties in the input parameters $D, M$, and $i$ and arrive at our final lower limit on $a_{*}$.

Another parameter of great interest is the scattering fraction, which measures the strength of the Compton component: $f_{\mathrm{SC}}=22.5 \% \pm 0.6 \%, 30.5 \% \pm 1.2 \%$, and $30.6 \% \pm 0.6 \%$ for SP1, SP2, and SP3, respectively. These values are high compared to values characteristic of the TD state $\left(f_{\mathrm{SC}} \lesssim 5 \%\right.$; Steiner et al. 2009b), which is the most favorable state for the measurement of black hole spin. These large values of $f_{\mathrm{SC}}$, as well as the uniform value of the power-law index $(\Gamma \sim 2.5)$, imply that for all three observations the source was in the SPL state (Remillard $\&$ McClintock 2006). Steiner et al. (2009b) have shown that one can obtain reliable values of the inner disk radius (and hence spin) in the SPL state for $f_{\mathrm{SC}} \lesssim 25 \%$ (see their Figure 1 ).

The luminosity of the disk component, $L / L_{\mathrm{Edd}} \approx 0.02$, is quite low and easily meets our data selection criterion, $L / L_{\text {Edd }}<0.3$ (McClintock et al. 2006). Correspondingly, the disk is geometrically very thin at all radii (the aspect ratio $h / r<0.05$; see Penna et al. 2010; Kulkarni et al. 2011). At the same time, the luminosity is sufficiently high that the spectral hardening factor $f$ is well determined by the data $(f \approx 1.6$ ).

Interestingly, for the pair of Chandra observations that are separated by just two days, the values of the fit parameters are quite similar (Table 2) with the notable exception of the column density: $N_{\mathrm{H}}=0.768 \pm 0.024 \mathrm{~cm}^{-2}$ for $\mathrm{SP} 2$ and $N_{\mathrm{H}}=0.687 \pm 0.010 \mathrm{~cm}^{-2}$ for SP3. We tested whether one can achieve a good fit with a single value of $N_{\mathrm{H}}$, by fitting the Chandra/RXTE spectra jointly, linking only the parameter $N_{\mathrm{H}}$. This linking increased the total chi-square by 41 , which corresponds to an F-test probability of $3.0 \times 10^{-9}$. This low probability implies a significant change in $N_{\mathrm{H}}$, which is likely due to absorption in the wind of the supergiant companion.

\footnotetext{
21 TBABS uses updated values for the photoelectric cross-sections and interstellar medium abundances and is an improved version of the familiar model PHABS. The choice of low-energy absorption model has a negligible affect on our results apart from the higher column density $(\sim 40 \%)$ that TВABS returns compared to PHABS.
} 
Table 2

Fit Results for Our Adopted Model ${ }^{\mathrm{a}}$

\begin{tabular}{|c|c|c|c|c|c|}
\hline Number & Model & Parameter & SP1 & $\mathrm{SP} 2$ & SP3 \\
\hline 1 & KERRBB2 & $a_{*}$ & $0.9985_{-0.0008}^{+0.0005 b}$ & $0.9999_{-0.0029}^{+0} \mathrm{~b}$ & $0.9999_{-0.0001}^{+0}{ }^{b}$ \\
\hline 2 & KERRBB2 & $\dot{M}$ & $0.115 \pm 0.004$ & $0.139 \pm 0.029$ & $0.122 \pm 0.011$ \\
\hline 3 & const & $\cdots$ & $1.000 \pm 0.002$ & $1.031 \pm 0.013$ & $0.971 \pm 0.004$ \\
\hline 4 & TBABS & $N_{\mathrm{H}}$ & $0.705 \pm 0.006$ & $0.768 \pm 0.024$ & $0.687 \pm 0.010$ \\
\hline 5 & SIMPLR & $\Gamma$ & $2.282 \pm 0.010$ & $2.499 \pm 0.012$ & $2.549 \pm 0.011$ \\
\hline 6 & SIMPLR & $f_{\mathrm{SC}}$ & $0.225 \pm 0.006$ & $0.305 \pm 0.012$ & $0.306 \pm 0.006$ \\
\hline 7 & KERRDISK & $E_{\mathrm{L}}$ & $6.56 \pm 0.09$ & $6.44 \pm 0.05$ & $6.49 \pm 0.04$ \\
\hline 8 & KERRDISK & $q$ & $2.82 \pm 0.02$ & $2.28 \pm 0.06$ & $2.29 \pm 0.07$ \\
\hline 9 & KERRDISK & $N_{\mathrm{L}}$ & $0.015 \pm 0.001$ & $0.032 \pm 0.002$ & $0.024 \pm 0.001$ \\
\hline 10 & KERRDISK & EW & 0.154 & 0.158 & 0.159 \\
\hline 11 & IREFLECT & $\mathrm{X}_{\mathrm{Fe}}$ & $5.34 \pm 0.15$ & $2.97 \pm 0.12$ & $3.63 \pm 0.14$ \\
\hline 12 & IREFLECT & $s$ & $0.98 \pm 0.04$ & $0.80 \pm 0.03$ & $0.93 \pm 0.03$ \\
\hline 13 & IREFLECT & $\xi$ & $153.1 \pm 15.7$ & $71.2 \pm 10.0$ & $62.0 \pm 8.4$ \\
\hline 14 & & $\chi_{v}^{2}$ & $1.24(561 / 454)$ & $1.28(371 / 290)$ & $1.18(723 / 614)$ \\
\hline 15 & & $f$ & 1.610 & 1.612 & 1.621 \\
\hline 16 & & $L / L_{\text {Edd }}$ & 0.018 & 0.026 & 0.023 \\
\hline 17 & Adopted $^{\mathrm{c}}$ & $a_{*}$ & $0.9985_{-0.0148}^{+0.0005}$ & $0.9999_{-0.0050}^{+0}$ & $0.9999_{-0.0116}^{+0}$ \\
\hline
\end{tabular}

Notes.

${ }^{a}$ For the model components given, the parameters from top to bottom are (1) spin parameter; (2) mass accretion rate in units of $10^{18} \mathrm{~g} \mathrm{~s}{ }^{-1}$; (3) detector normalization constant relative to RXTE PCU2; (4) hydrogen column density in units of $\mathrm{cm}^{-2}$; (5) photon power-law index $\Gamma$; (6) scattering fraction $f_{\mathrm{SC}}$; (7) central line energy in $\mathrm{keV}$; (8) emissivity index $q$; (9) line flux in units of photons $\mathrm{cm}^{-2} \mathrm{~s}^{-1}$; (10) equivalent width of line in $\mathrm{keV}$; (11) iron abundance relative to solar; (12) reflection scaling factor $s$; (13) ionization parameter $\xi$; (14) Reduced chi-square, total chi-square, and degrees of freedom, respectively; (15) spectral hardening factor $f$; and (16) Eddington-scaled disk luminosity, where $L_{\text {Edd }} \approx 1.9 \times 10^{39} \mathrm{erg} \mathrm{s}^{-1}$ for Cygnus X-1. Unless otherwise indicated, the uncertainties quoted here and throughout the paper are at the $1 \sigma$ level of confidence.

b The physical maximum value of the spin parameter $a_{*}$ is unity and for the XSPEC model KERRBB 2 it is 0.9999 . The very small errors quoted here are purely the uncertainties due to counting statistics, which result from fitting our adopted model to the X-ray data.

${ }^{c}$ Final adopted values for the spin parameter and their uncertainties. The $1 \sigma$ uncertainties are calculated based on the $3 \sigma$ lower limits on $a_{*}$ shown in Figure 4. These results fold in the uncertainties in $D, M, i$, and the absolute flux calibration via our Monte Carlo analysis (see Section 6).

Indeed, one expects such absorption in a wind to be larger for SP2 at orbital phase 0.24 than for SP3 at phase 0.61 , as observed (Table 1; Bałucińska-Church et al. 2000).

\section{ROBUSTNESS OF SPIN ESTIMATES}

In this section, we consider a number of factors that might affect our results and find that none of them is significant. We consider the effects of (1) excluding the $\mathrm{Fe} \mathrm{K} \alpha$ line and edge features from the fits, (2) including HEXTE data and extending the fits to $150 \mathrm{keV}$, (3) using REFLIONX to model the reflection component, and (4) substituting $\alpha=0.01$ for our fiducial value of $\alpha=0.1$ and varying the metallicity. As shown below, factors (1) and (2) have negligible effects on our results, and (3) and (4) have slight upward effects on $a_{*}$, which implies that the extreme values reported in Table 2 are conservative lower limits. Finally (Section 5.5), we explore the effects on our results of artificially relaxing the spin parameter away from its extreme, limiting value by varying the parameters of KERRBB2; we find that the input parameters $D, M$, and $i$ have to be driven far from their fiducial values in order to obtain a spin value as low as $a_{*}=0.9$.

\subsection{Effect of Iron Line and Edges}

For all three spectra, we refitted the data excluding the energy range 5.0-10.0 keV while omitting the component KERRDISK. This excised energy range contains the relativistically broadened $\mathrm{Fe} \mathrm{K} \alpha$ line and edge, as well as a significant residual feature near $9 \mathrm{keV}$ (Figure 3). For all three spectra, we find that our results are essentially unchanged, apart from small shifts in the parameters of the reflection component. The results for spectrum SP1 (only) are shown in Table 3 (Case 2) where they are compared to our standard results (Case 1). Thus, we find that the values of the spin parameter returned by the fits are completely determined by the temperature and luminosity of the thermal component and are unaffected by the presence of the line. This conclusion is reasonable given that the line is a minor feature with an equivalent width of $\approx 0.15 \mathrm{keV}$ or only $\approx 0.015 \mathrm{keV}$ relative to the continuum at the peak of the thermal component (see Figure 3).

\subsection{Effect of Extending the Bandwidth to $150 \mathrm{keV}$}

In Section 2, we asserted that the coverage of the PCA to $45 \mathrm{keV}$ was sufficient to adequately constrain the power-law and reflection components. We now demonstrate that this is true by refitting the data for SP1 (only) while including the HEXTE data spanning the energy range $20 \mathrm{keV}$ to $150 \mathrm{keV}$ (where the source counts are negligible). For the HEXTE data, we do not add a systematic error to the count rates. However, we do correct the detector response to the Crab spectrum of Toor \& Seward (see Section 2) using the value of the Crab's photon index as measured using HEXTE: ${ }^{22} \Gamma=1.93 \pm 0.003$. The slope correction is $\Delta \Gamma_{\mathrm{TS}}=-0.17$. The results obtained using the HEXTE data, which are given in Table 3 (Case 3), are almost identical to our standard results for SP1 (Case 1). This is not surprising because the PCA coverage to $45 \mathrm{keV}$ is more than adequate to determine the slope of the power-law component,

\footnotetext{
22 http://web.mit.edu/iachec/IACHEC_2_talks/IACHEC_II_suchy.pdf
} 
Table 3

Effects of Excluding the Fe K $\alpha$ Line and Increasing Bandwidth (SP1 Only) ${ }^{\mathrm{a}}$

\begin{tabular}{llcccr}
\hline \hline Number & Model & Parameter & Case 1 & Case 2 & Case 3 \\
\hline 1 & KERRBB2 & $a_{*}$ & $0.9985_{-0.0008}^{+0.0005}$ & $0.9983_{-0.0037}^{+0.0005}$ & $0.9984_{-0.0003}^{+0.0001}$ \\
2 & KERRBB2 & $\dot{M}$ & $0.115 \pm 0.004$ & $0.115 \pm 0.007$ & $0.115 \pm 0.003$ \\
3 & const & $\ldots$ & $1.000 \pm 0.002$ & $0.999 \pm 0.002$ & $1.000 \pm 0.002$ \\
4 & TBABS & $N_{\mathrm{H}}$ & $0.705 \pm 0.006$ & $0.708 \pm 0.009$ & $0.706 \pm 0.005$ \\
5 & SIMPLR & $\Gamma$ & $2.282 \pm 0.010$ & $2.288 \pm 0.013$ & $2.287 \pm 0.008$ \\
6 & SIMPLR & $f_{\mathrm{SC}}$ & $0.225 \pm 0.006$ & $0.221 \pm 0.010$ & $0.221 \pm 0.005$ \\
7 & KERRDISK & $E_{\mathrm{L}}$ & $6.56 \pm 0.09$ & $0.00 \pm 0.00$ & $6.56 \pm 0.05$ \\
8 & KERRDISK & $q$ & $2.82 \pm 0.02$ & $2.82 \pm 0.23$ & $2.81 \pm 0.02$ \\
9 & KERRDISK & $N_{\mathrm{L}}$ & $0.015 \pm 0.001$ & $0.000 \pm 0.000$ & $0.015 \pm 0.001$ \\
10 & KERRDISK & $\mathrm{EW}$ & 0.154 & $\ldots$ & 0.153 \\
11 & IREFLECT & $\mathrm{X}_{\mathrm{Fe}}$ & $5.34 \pm 0.15$ & $3.93 \pm 0.52$ & $4.72 \pm 0.12$ \\
12 & IREFLECT & $s$ & $0.98 \pm 0.04$ & $1.00 \pm 0.10$ & $0.94 \pm 0.03$ \\
13 & IREFLECT & $\xi$ & $153.1 \pm 15.7$ & $189.6 \pm 23.7$ & $153.3 \pm 11.6$ \\
\hline 14 & & $\chi_{v}^{2}$ & $1.24(561 / 454)$ & $0.82(297 / 361)$ & $1.25(616 / 493)$
\end{tabular}

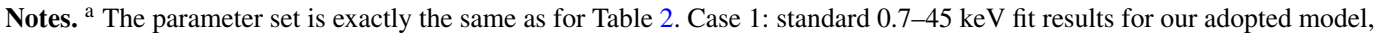
which are copied from column SP1 in Table 2. Case 2: fit to SPI over the range 0.7-45 keV excluding 5.0-10.0 keV (i.e., excluding the $\mathrm{Fe} \mathrm{K} \alpha$ line and Fe absorption edge). The value of $q$ is that returned by KERrCONV. Case 3: fit to SP1 including HEXTE data that covers the full energy range from 0.7 to $150 \mathrm{keV}$.

and the reflection component is quite weak and dying rapidly at $45 \mathrm{keV}$ (Figure 3).

\subsection{Effect of Using a Different Reflection Model}

We replace IREFLECT $\otimes$ SIMPLC+KERRDISK with REFLIONX ${ }^{23}$ (Ross \& Fabian 2005), which is widely used in measuring spin via the $\mathrm{Fe} \mathrm{K} \alpha$ line. The merit of this alternative reflection model is that it self-consistently calculates the line feature and the reflection component, whereas IREFLECT models only the absorption edges. The downside of REFLIONX is its description of the seed photon distribution as a simple power law, which unphysically diverges at low energies (for further comparison of the two models see Steiner et al. 2011). We include relativistic blurring by convolving REFLIONX with KERRCONV. Compared to the results given in Table 2, the values of the parameters returned by the fits are generally different, although reasonable, and the fits are somewhat poorer $\left(\chi_{v}^{2}=1.35-1.45\right)$. Importantly, the effects on the spin parameter are very small: the value of $a_{*}$ increases slightly for SP1 (0.9985-0.9999) and is unaffected for SP2 and SP3, which are at their maximum values.

\subsection{Effect of Varying the Viscosity Parameter and Metallicity}

We refitted the spectra using $\alpha=0.01$ in place of our fiducial value, $\alpha=0.1$. Again, the spin parameter for SP1 increases slightly (0.9985-0.9988), while the values for SP2 and SP3 remain unchanged. Concerning possible metallicity effects, we do not have nonsolar-metallicity table models for computing spectral hardening for such extreme values of spin. However, for three sources (M33 X-7, Liu et al. 2008; LMC X-1, Gou et al. 2009; and A0620-00, Gou et al. 2010), we have found that substantial changes in metallicity produce very small changes in $a_{*}$. For example, reducing the metallicity from solar to a tenth solar decreases the spin parameter of a highspin black hole like LMC X-1 $\left(a_{*}=0.938 \pm 0.020\right)$ by only $\Delta a_{*}=0.001$. The effect of this same change in metallicity for the slowly-spinning black hole A0620-00 $\left(a_{*}=0.135 \pm\right.$ 0.029 ) is larger, but it is still quite small: $\Delta a_{*}=0.014$. (The

\footnotetext{
23 The model can be downloaded at

http://heasarc.gsfc.nasa.gov/docs/xanadu/xspec/models/reflion.html.
}

small errors on $a_{*}$ quoted here come directly from fitting the Xray spectra, and they do not take into account the uncertainties in $D, M$, and $i$, which dominate the error budget.) We note that the super-solar iron abundances implied by our fits using the reflection models, IREFLECT (Table 2) and PEXRIV (Appendix B), suggest that the metallicity of the Cygnus X-1 system is possibly enhanced. Accounting for the supersolar abundances will result in a slightly increased estimate of $a_{*}$, so our conclusions are robust to enhanced metallicity.

\subsection{Relaxing the Spin Parameter}

We now describe three technical exercises that artificially examine the effects of varying three parameters of KERRBB2, namely, $a_{*}, D$, and the normalization constant $N_{\mathrm{K}}$ (which we have elsewhere fixed to unity, as is appropriate when $D, M$, and $i$ are specified). Our motivation is to examine the consequences of relaxing the spin parameter away from the extreme values returned by the fits (Table 2).

First, for spectrum SP1 only, we leave $N_{\mathrm{K}}$ fixed at unity and allow the distance to vary (keeping $M$ and $i$ fixed at their fiducial values). For our parallax distance of $D=1.86 \mathrm{kpc}$, we of course have our standard result, $a_{*}=0.9985$ (Table 2). We now arbitrarily and successively fix the spin parameter at two lower values, $a_{*}=0.95$ and $a_{*}=0.90$, and refit spectrum SP1. The best-fit values of $D$ are then substantially greater than our measured value of $1.86 \mathrm{kpc}: 2.43 \pm 0.03 \mathrm{kpc}$ and $2.78 \pm$ $0.02 \mathrm{kpc}$, respectively. Meanwhile, the corresponding values of reduced chi-square are respectively 1.34 and 1.40 , which are significantly greater than our standard value of 1.24 (Table 2). Thus, the best fit is achieved for the observed and extreme value of spin.

Secondly and alternatively, we obtain similar results by varying $N_{\mathrm{K}}$ while leaving the distance fixed at its fiducial value of $1.86 \mathrm{kpc}$. For the same pair of forced values of the spin parameter given above $(0.95$ and 0.90$)$, we find for spectrum SP1 that the fitted values of $N_{\mathrm{K}}$ are about half the standard value of unity: $0.65 \pm 0.01$ and $0.45 \pm 0.01$, respectively (where a smaller value corresponds to a weaker thermal component). Meanwhile, we find values of reduced chi-square that are very similar to those given in the previous example, with values that 


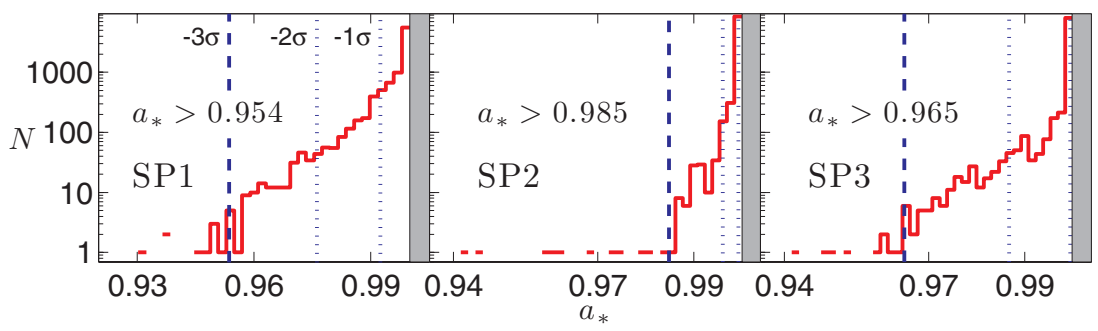

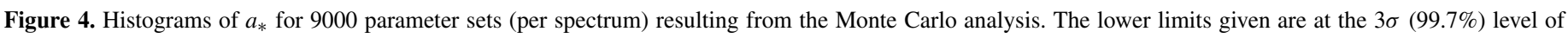
confidence.

(A color version of this figure is available in the online journal.)

increase as the spin parameter decreases. That is, we again find that the best fit is achieved for the observed and extreme value of spin.

In a final experiment, we assess the consistency of our spin values for the three spectra, which cannot be adequately judged by considering the extreme values given in Table 2 . We make this assessment by artificially setting $N_{\mathrm{K}}=0.5$ in our adopted model and refitting the three spectra. In this way, we find best-fit spin values that are in reasonable agreement: $a_{*}=0.937_{-0.001}^{+0.001}$ for SP1, $a_{*}=0.934_{-0.020}^{+0.021}$ for SP2, and $a_{*}=0.953_{-0.006}^{+0.007}$ for SP3.

\section{COMPREHENSIVE ERROR ANALYSIS}

In all previous work on measuring spin, we have found that the statistical uncertainty in the estimated spin parameter due to the X-ray data analysis is small compared to that due to observational uncertainties in $D, M$, and $i$. Based on our GRMHD simulations of thin disks (Shafee et al. 2008; Penna et al. 2010), we have likewise found that the uncertainties in these three input parameters dominate over the errors resulting from our use of the analytic Novikov-Thorne model, which assumes a razor-thin disk. In the case of Cygnus X-1, these model errors are especially small because of the extreme spin of the black hole and the low luminosity of the disk. Spin estimates obtained by fitting mock spectra of simulated GRMHD disks indicate that for an inclination $i=30^{\circ}$, which is very near the inclination of Cygnus X-1 $(i=27.1)$, the Novikov-Thorne thin-disk model overestimates the spin parameter by only $\Delta a_{*}=0.007$ and 0.005 for spins of 0.90 and 0.98 , respectively (see Table 1 in Kulkarni et al. 2011). Furthermore, these errors are significantly overestimated because they were computed for disks that are far more luminous $\left(L / L_{\text {Edd }} \sim 0.5\right)$, and hence thicker, than that of Cygnus X-1 $\left(L / L_{\mathrm{Edd}} \approx 0.02\right) .{ }^{24}$ In our error analysis, we neglect this small model error.

With the exception of our recent spin measurement of XTE J1550-564 (Steiner et al. 2011), in earlier work we have neglected the uncertainty in the luminosity due to the $\sim 10 \%$ uncertainty in the flux of the Crab (Toor \& Seward 1974), an error that uniformly shifts all of our spin measurements either up or down. For the spin of Cygnus X-1, the effect of the uncertainty in the absolute flux calibration is very comparable to the $6 \%$ uncertainty in $D$ (which is equivalent to a $12 \%$ uncertainty in the measurement of flux). We have therefore included in our error budget the $10 \%$ uncertainty in flux (which we approximate as an uncertainty in the distance of $0.1 \mathrm{kpc}$ ) by simply combining the distance and flux-calibration errors in quadrature, thereby inflating the actual $0.120 \mathrm{kpc}$ distance uncertainty to $0.156 \mathrm{kpc}$.

\footnotetext{
${ }^{24}$ It is computationally very challenging to simulate thinner disks.
}

Thus, the final error we report for $a_{*}$ includes the uncertainties in $D, M, i$ and the uncertainty in the absolute flux calibration. Taken together, these four sources of uncertainty totally dominate the error budget.

In order to determine the error in $a_{*}$ due to the combined uncertainties in $D, M$, and $i$, we performed Monte Carlo simulations using the Odyssey computing cluster at Harvard University. The latter two parameters are not independent. They are related through the expression for the mass function: $f(M) \equiv M^{3} \sin ^{3} i /\left(M+M_{\mathrm{opt}}\right)^{2}=0.263 \pm 0.004 M_{\odot}$, where $M_{\text {opt }}=19.16 \pm 1.90 M_{\odot}$ is the mass of the secondary star and the value of the mass function was evaluated using a K-velocity of $76.79 \pm 0.41 \mathrm{~km} \mathrm{~s}^{-1}$ and an orbital period of $P=5.599829$ days (Orosz et al. 2011).

In the analysis, we assumed that the value of the mass function, the inclination, and the mass of the secondary are normally and independently distributed, and we computed the mass of the black hole using the values of these quantities, which are given above. We conservatively fixed the viscosity parameter at its baseline value, $\alpha=0.1$ (using $\alpha=0.01$ increases $a_{*}$; see Section 5.4). Specifically, we (1) generated 9000 parameter sets for $D, i, M_{\mathrm{opt}}$, and $f(M)$; (2) solved for $M$ for a given triplet of values of $i, M_{\mathrm{opt}}$, and $f(M)$; (3) computed for each set the look-up table for the spectral hardening factor $f$ using the model BHSPEC; and (4) obtained $a_{*}$ by fitting our adopted model to the spectra. The final histogram distributions for our three spectra are shown in Figure 4. Consulting these histograms, we see that the spin estimate is lowest for SP1, and we conservatively base our final result on this spectrum, thereby concluding that $a_{*}>0.95$ at the $3 \sigma$ level of confidence (see Figure 4 and the bottom line of Table 2).

We note the following two caveats. First, the power-law component is strong relative to the thermal component, which decreases the reliability of the continuum-fitting method (Steiner et al. 2010, 2011). Second, as we discuss in Section 7.4, the continuum-fitting method assumes that the spin vector of the black hole is aligned with the orbital angular momentum vector.

\section{DISCUSSION}

Our wide-ranging discussion covers seven topics. (1) We first confront the challenge posed by a grossly discrepant spin result that was obtained using the Fe-line method (while noting a concordant Fe-line result that appeared very recently) and (2) a second discrepant result obtained using a quasiperiodic oscillation (QPO) method. (3) We next consider a disfavored dynamical model that implies a less extreme value of spin. (4) The fundamental assumption of the continuumfitting method, namely, the alignment of the spin and orbital vectors, is then considered. (5) The extreme spin of the black 
hole is reconciled with the low temperature of its accretion disk. (6) We find no evidence in our Chandra HETG spectra for warm absorbing gas, and we show that, if it were present, its effects on our spin estimates would be negligible. (7) Finally, we discuss some consequences of the extreme spin of Cygnus X-1, and we argue that this spin is chiefly natal in origin.

\subsection{Measurement of Spin via the Fe Ka Method}

Based on an analysis of the $\mathrm{Fe} \mathrm{K} \alpha$ line profile, Miller et al. (2005) found marginal evidence for high spin using the same $A S C A$ spectrum of Cygnus X-1 that we use. Subsequently, however, Miller et al. (2009) reported a spin of $a_{*}=0.05 \pm 0.01$ based on an analysis that combines $\mathrm{Fe} \mathrm{K} \alpha /$ reflection models and continuum models, including KERRBB, a relativistic disk model similar to the one we use. That is, the authors jointly applied the continuum-fitting and $\mathrm{Fe} \mathrm{K} \alpha$ methods and fitted simultaneously for a single value of $a_{*}$. The data analyzed in this case were a pair of $0.5 \mathrm{ks} X M M-N e w t o n / E P I C-p n$ spectra obtained in the "burst" timing mode.

While it is not possible for us to account in detail for the gross difference between the near-zero spin reported by Miller et al. (2009) and our near-extreme value, we note the following. First, the round values of $D, M$, and $i$ that Miller et al. used as input to KERRBB differ significantly from ours, and these differences all serve to drive the spin-down, e.g., using their values of these parameters and our spectrum SP1, we find $a_{*}=0.74 \pm 0.01$. Second, and in addition, Miller et al. fitted for the KERRBB normalization constant, obtaining $N_{\mathrm{K}}=0.31$, whereas the standard procedure, which we follow, is to set $N_{\mathrm{K}}$ to unity when $D, M$, and $i$ are fixed. Fitting SP1 using $N_{\mathrm{K}}=0.31$ (and the values of $D, M$, and $i$ adopted by Miller et al.), we find that the spin drops from $a_{*}=0.78$ to the retrograde value $a_{*}=-0.53$ (and the fit is poor, $\chi_{v}^{2}=2.01$.)

The near-zero spin result of Miller et al. (2009) is further called into question because no data above $10 \mathrm{keV}$ were used. This lack of high energy coverage, in the presence of a strong Compton component, seriously compromises results obtained using the continuum-fitting method, as we stress at the outset of Section 2 (and as can be deduced by an examination of Figure 3). Likewise, results obtained using the $\mathrm{Fe} \mathrm{K} \alpha /$ reflection method are compromised by the failure to observe the Compton reflection hump around 20-30 keV (e.g., see Larsson et al. 2008).

Very recently, after posting our paper on the astro-ph archive and while revising it for resubmission, a paper appeared reporting another estimate of the spin of Cygnus X-1 via an analysis of the Fe K $\alpha$ profile (Duro et al. 2011). Assuming that the emissivity profile of the disk can be described by a single power law with index 3.0, these authors conclude (p. 1) that "the black hole is close to rotating maximally," which is in agreement with our result. We note that Duro et al. do not discuss Miller et al. (2005, 2009) or mention the near-zero spin result reported in the latter paper.

\subsection{Measurement of Spin via a QPO Model}

Based on an analysis of low-frequency (0.01-25 Hz) QPOs, Axelsson et al. (2005) obtained a spin for Cygnus X-1 of $a_{*}=0.49 \pm 0.01$ for $M=8 M_{\odot}$. Their result is based on the relativistic precession model of Stella et al. (1999). In this model, the QPO is produced as a result of emission from an orbiting bright spot that is undergoing relativistic nodal and periastron precessions in a slightly tilted and eccentric orbit. The spin parameter is predicted to vary with mass as $a_{*} \propto M^{-1 / 5}$ (see Equation (4) in Axelsson et al. 2005), and therefore the corrected value of spin is $a_{*}=0.43$ for our adopted black hole mass $M=14.8 M_{\odot}$. The large discrepancy between this moderate value of spin and the extreme value we find may be a consequence of a fundamental assumption of their model, namely, that the black hole is rotating slowly $\left(a_{*} \ll 1\right)$. On the other hand, the precession model, with its assumption of geodesic motion, may not apply in this instance.

\subsection{An Alternative Dynamical Model}

In Orosz et al. (2011), results are presented for four dynamical models, Models $\mathcal{A}-D$. We disregard Models $\mathcal{A}$ and $\mathcal{B}$, which assume a circular orbit, because these models give poor fits compared to the eccentric orbit models $\left(\Delta \chi^{2}>50\right)$ and because there is clear evidence that the orbit is eccentric. Throughout this paper, we have used $M=14.8 \pm 1.0 M_{\odot}$ and $i=27.1 \pm 0.8$ from Model $\mathcal{D}$, an asynchronous model with a rotational frequency for the O-star that is $40 \%$ greater than the orbital frequency. As an alternative to $\operatorname{Model} \mathcal{D}$, we now consider Model $\mathcal{C}$, which assumes synchronous rotation. Model $\mathcal{C}$ gives a poorer fit to the data $\left(\Delta \chi^{2} \approx 13\right)$, and it results in disharmony between the light curve and velocity data on the one hand, and the radius and rotational velocity of the O-star on the other (see Table 1 in Orosz et al. 2011). Because of this disharmony, the uncertainties in $M$ and $i$ for Model $\mathcal{C}$ are significantly larger than those for our favored model, although the central values of these parameters differ only modestly: $M=15.8 \pm 1.8 M_{\odot}$ and $i=28.5 \pm 2.2$. Using these values for Model $\mathcal{C}$ and spectrum SP1 (which gives the lowest value of spin), we repeated our error analysis (Section 6) and obtained the following $3 \sigma$ lower limit on the spin parameter: $a_{*}>0.92$.

\subsection{Alignment of Spin and Orbital Angular Momentum}

There remains one uncertainty that calls into question the reliability of the continuum-fitting method, namely, whether the inner X-ray emitting portion of the disk (which will align with the black hole's spin axis) is aligned with the binary orbital plane. For a discussion of this question, see Section 2.2 in $\mathrm{Li}$ et al. (2009). As an extension of this discussion, we note the following. First, recent population synthesis studies predict that the majority of systems will have rather small $\left(\lesssim 10^{\circ}\right)$ misalignment angles (Fragos et al. 2010). Second, in the case of Cygnus $X-1$, there is reason to believe that the misalignment angle is especially small because of the binary system's low peculiar velocity, which indicates that the system did not experience a large "kick" when the black hole formed (Mirabel \& Rodrigues 2003; Reid et al. 2011). As demonstrated in Figure 5, even if there exists a misalignment angle as large as, e.g., $16^{\circ}$, the spin value is still $>0.95$.

\subsection{Low Disk Temperature Compatible with Extreme Spin}

One might expect that the accretion disk of a fast-spinning black hole like Cygnus X-1 would be hot $\left(T_{\text {in }}>1 \mathrm{keV}\right)$ because its inner edge is relatively close to the event horizon and deep in the gravitational potential well. Two principal factors are responsible for the depressed disk temperature $\left(T_{\text {in }} \approx 0.5 \mathrm{keV}\right.$; Table 7). The first of these is the low rate of mass accretion through the disk, which is manifested by its low luminosity, only 


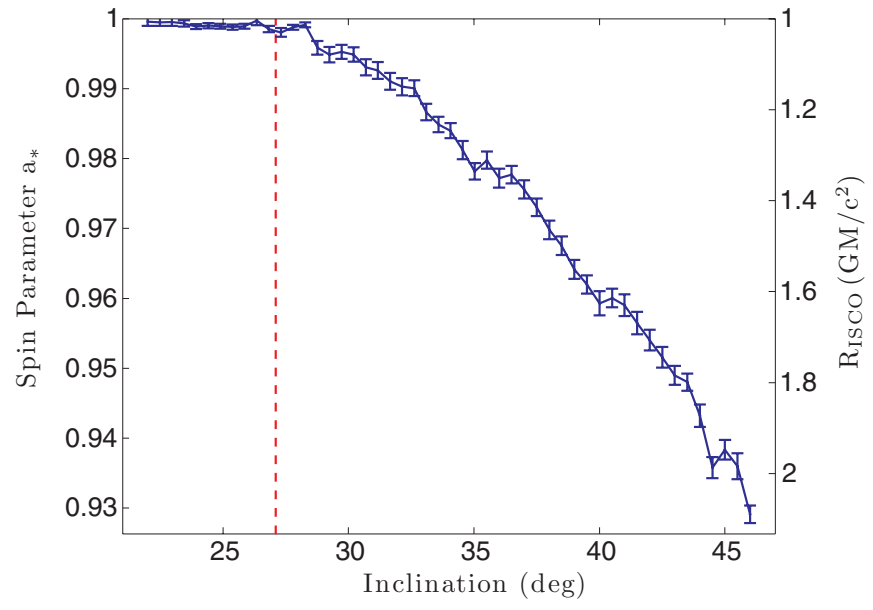

Figure 5. Effect on the spin parameter of varying the inclination angle $i$ (for SP1 only) for fixed values of our adopted parameters: $D=1.86 \mathrm{kpc}$ and $M=14.8 M_{\odot}$. The best-fit value of inclination $(i=27.1)$ is indicated by the dashed line. While the spin parameter in this figure varies modestly from 0.93 to unity, the ISCO radius - the quantity that we actually measure - can be seen to change by a factor of two (Bardeen et al. 1972). As an extreme example, as $a_{*}$ increases from 0.98 to 0.99 , the fractional change in the ISCO radius is 10 times the fractional change in the spin parameter. This "saturation" of the spin parameter near unity is the reason that the statistical uncertainties given in Table 2 are so small.

(A color version of this figure is available in the online journal.)

$\approx 2 \%$ of Eddington (Table 2). The second is that relativistic effects (e.g., beaming and light bending) are muted for low disk inclinations. Using our fiducial values of $D, M$, and $i$, and fixed values of the fit parameters taken from Table 2, we used KERRBB2 to simulate a pair of spectra, one for the inclination of Cygnus X-1, $i=27.1$, and the other for $i=66.0$ (which is the inclination of GRS 1915+105; McClintock et al. 2006). The luminosities of the two spectra are the same, $L / L_{\text {Edd }} \approx 0.018$, while the disk temperatures are $T_{\text {in }}=0.45 \mathrm{keV}$ and $0.86 \mathrm{keV}$, respectively. Thus, in this comparison, the temperature of the low-inclination disk is depressed by nearly a factor of two because the relativistic effects are weak.

\subsection{Effects of a Warm Absorber}

Although careful modeling of warm absorbers (WAs) is usually crucial in determining the spins of supermassive black holes via the Fe $\mathrm{K} \alpha$ method (e.g., Brenneman \& Reynolds 2006), we find that the effects of WAs are unimportant in estimating the spin of Cygnus X-1 via the continuum-fitting method. We examined all of the available soft-state Chandra HETG spectra of Cygnus X-1 (ObsIDs 2741, 2742, 2743, 12313/SP2, and $12314 / \mathrm{SP} 3)$ at $E<1 \mathrm{keV}$, and we find no evidence for the blend of absorption lines near $0.76 \mathrm{keV}$ (due, e.g., to $\mathrm{Fe} \mathrm{X}-\mathrm{Fe}$ $\mathrm{XV}, \mathrm{O}$ VII, and O VIII), which is a telltale signature of a WA. (The line WAs produce above $1 \mathrm{keV}$ are mostly discrete and weaker and therefore have an accordingly much smaller effect on the continuum shape and our spin estimates.)

Our preliminary analysis of the low-energy portion $(E<$ $0.8 \mathrm{keV}$ ) of a $50 \mathrm{ks}$ hard-state Chandra HETG spectrum (Hanke et al. 2009) did reveal the presence of a single WA. Its two most relevant parameters are its column density, $N_{\mathrm{H}}=$ $7.2 \times 10^{20} \mathrm{~cm}^{-2}$, and ionization parameter, $\xi=44$. (Its turbulent broadening and redshift are, respectively, $v=47.6 \mathrm{~km} \mathrm{~s}^{-1}$ and $z=-0.00086$; we assume solar abundances.) This tenuous WA does not affect the continuum shape, but it does produce line blends, the strongest of which is centered at $0.76 \mathrm{keV}$. Although such a WA is not present in soft-state spectra, we nevertheless tested its effects by reanalyzing SP1, SP2, and SP3 by introducing the additional model component WARMABS, fixing its parameters to the values given above; we obtained values of spin that are essentially identical to those given in Table 2. In one further test, we likewise reanalyzed our three spectra including a second thicker and hotter WA that generates a complex of absorption lines between 0.9 and $1.0 \mathrm{keV}$ $\left(N_{\mathrm{H}}=1.0 \times 10^{21} \mathrm{~cm}^{-2} ; \xi=300 ; v=100 \mathrm{~km} \mathrm{~s}^{-1}\right.$; $z=-0.00086)$. Again, the effects on our spin estimates are negligible.

\subsection{Consequences of Extreme Spin and Its Origin}

The spin of Cygnus X-1 is extraordinarily high, placing it in the company of the microquasar GRS $1915+105$, estimated using the continuum-fitting method (McClintock et al. 2006; Blum et al. 2009), and the supermassive black hole in MCG-6-30-15, estimated using the Fe-line method (Brenneman \& Reynolds 2006). The spins of both of these black holes are reported to exceed $a_{*}=0.98$. As Penrose (1969) first demonstrated, the enormous "flywheel" energy of a fast-spinning black hole can in principle be tapped. For Cygnus X-1, the potentially tappable energy is $>2.8 M_{\odot} c^{2}=5.0 \times 10^{54} \mathrm{erg}$ (Christodoulou \& Ruffini 1971); in comparison, the energy radiated by the Sun over its entire $\sim 10$ billion year lifetime is $\lesssim 0.001 M_{\odot} c^{2}$. It has been widely suggested that spin-down energy powers the relativistic jets observed for at least some quasars and microquasars (Blandford \& Znajek 1977), such as GRS 1915+105 (Mirabel \& Rodríguez 1994).

As the spin $a_{*}$ of a black hole approaches unity, the radius of its ISCO approaches the radius of the event horizon $R_{\mathrm{EH}}$ (Bardeen et al. 1972). For Cygnus X-1, with spin $a_{*}>0.95$ and $M=14.8 M_{\odot}, R_{\mathrm{ISCO}}<42 \mathrm{~km}$ while $R_{\mathrm{EH}}<29 \mathrm{~km}$. The Keplerian velocity of the gas at the ISCO is approximately half the speed of light, and its orbital frequency is $>598 \mathrm{~Hz}$. Meanwhile, the frequency of rotation of the black hole itself, which is the spin frequency of space time at its horizon, is $>790 \mathrm{~Hz}$ (the maximal spin frequency for $a_{*}=1$ is $1091 \mathrm{~Hz}$ ).

What is the origin of the spin of Cygnus X-1? Was the black hole born with its present spin or was it torqued up gradually by the gas it has accreted over its lifetime? To achieve a spin of $a_{*}>0.95$ via disk accretion, an initially nonspinning black hole must accrete $>7.3 M_{\odot}$ from its donor (Bardeen 1970; King $\&$ Kolb 1999) in becoming the $M=14.8 M_{\odot}$ that we observe today. However, to transfer this much mass even at the maximum (Eddington-limited) accretion rate requires $>31$ million years, ${ }^{25}$ whereas the age of the binary system is between 4.8 and 7.6 million years $^{26}$ (Wong et al. 2011). (Even for $a_{*}>0.92$ obtained for our less probable model, the accretion timescale is $>25$ million years.) Thus, it appears that the spin of Cygnus X-1 must be chiefly natal (also, see Axelsson et al. 2011; Wong et al. 2011), although possibly the high spin could be achieved during

\footnotetext{
25 The corresponding estimates of spin-up time we reported for both M33 X-7 (Liu et al. 2008, 2010) and LMC X-1 (Gou et al. 2009) are incorrect because for the efficiency $\eta$ (of converting mass to radiant energy) we used a constant value, $\eta=1$. However, $\eta$ gradually increases as the black hole spins up, starting at $a_{*}=0$ with $\eta=0.06$ to $\eta=0.13\left(a_{*}=0.84\right)$ for M33 X-7 and $\eta=0.17\left(a_{*}=0.92\right)$ for LMC X-1. For M33 X-7 and LMC X-1, the correct limits on the spin-up timescales are $>17$ and $>25$ million years, respectively, while the respective ages of the systems are $\lesssim 3$ and $\lesssim 5$ million years.

26 A 7.6 million-year-old system accreting at the maximum rate would only achieve a spin of 0.59 .
} 
Table 4

The Three Nonrelativistic Models

\begin{tabular}{ll}
\hline \hline Number & Model \\
\hline NR1 & CRABCOR $^{*}$ CONST $^{*}$ TBABS(DISKBB+COMPBB+PEXRIV+GAUSSIAN) \\
NR2 & CRABCOR $^{*}$ CONST $^{*}$ TBABS(KDBLUR $\otimes($ DISKBB+COMPBB+PEXRIV)+LAOR) \\
NR3 & CRABCOR $^{*}$ CONST $^{*}$ TBABS(KDBLUR $\otimes($ SIMPLR $\otimes$ DISKBB+PEXRIV)+LAOR) \\
\hline
\end{tabular}

a short-lived evolutionary phase of hypercritical mass accretion (Moreno Méndez 2011).

\section{CONCLUSION}

Based on our favored dynamical model, we find an extreme value of spin for the black hole primary in Cygnus $\mathrm{X}-1$ : $a_{*}>0.95$ at the $3 \sigma$ level of confidence. For a less probable (synchronous) dynamical model, the spin is still high: $a_{*}>0.92$ $(3 \sigma)$. For both of these strong limits, we include the customary uncertainties in the input parameters $D(6 \%), M(7 \%)$, and $i$ $( \pm 0.8)$, and we also include the uncertainty in the calibration of the absolute flux via the Crab (10\%). These four sources of uncertainty totally dominate the error budget.

Our measurement of spin is determined solely by the properties of the thermal component and is unaffected by the presence of the relatively faint $\mathrm{Fe} \mathrm{K} \alpha$ line. Nevertheless, we have modeled this relativistically broadened line feature carefully in order to achieve good fits over the full range of energies we consider, which in our analyses variously extends from $0.5 \mathrm{keV}$ to $150 \mathrm{keV}$. The extreme spin we find for this black hole is based on an analysis of three broadband spectra that are each capable of constraining the soft thermal component, the hard Compton component, and the reflected component. By considering several different models and performing a number of tests on our results, we have demonstrated that the extreme spin we find is insensitive to the details of our analysis.

We thank an anonymous referee for very helpful and constructive comments. We are grateful to Director $\mathrm{H}$. Tanananbaum and Project Scientist T. Strohmayer for granting us, respectively, Chandra and RXTE observing time. We thank H. Marshall, M. Nowak, and N. Schulz for help in planning the Chandra observations, and M. Hanke, M. Nowak, and J. Wilms for discussions on X-ray data analysis. The research has made use of data obtained from the High Energy Astrophysics Science Archive Research Center (HEASARC) at NASA/Goddard Space Flight Center. L.G. thanks the Harvard FAS Sciences Division Research Computing Group for their technical support on the Odyssey cluster. J.E.M. acknowledges support from NASA grants DD0-11049X, DD1-12054X, and NNX11AD08G, and the Smithsonian Endowment Funds.

\section{APPENDIX A}

\section{PRELIMINARY NONRELATIVISTIC ANALYSIS}

With DISKBB as the principal component, we analyzed our three spectra in turn using three composite models of increasing sophistication, the Models NR1-NR3 listed in Table 4. In addition to DISKBB, each model includes (1) a Compton powerlaw component, either СОМРBв (Nishimura et al. 1986) or SIMPLR (Steiner et al. 2009b, 2011) convolved with DISKBB; and (2) a reflection component PEXRIV (Magdziarz \& Zdziarski 1995), ${ }^{27}$ which models the absorption edges, plus an Fe $\mathrm{K} \alpha$ emission-line feature GAUSSIAN or LAOR (Laor 1991). As in the case of our adopted model, also included are the three pre-factors CRABCOR, CONST, and TBABS (see Section 3.2). In the case of Models NR2 and NR3, relativistic blurring effects are included using KDBLUR (Laor 1991).

Model NR1: Cui et al. (1998) analyzed spectrum SP1 using this model, and we do likewise. For the thermal component, our values of the parameters (disk temperature $T_{\text {in }}$ and inner disk radius $R_{\text {in }}$ ) are quite similar to those found by Cui et al.: respectively, $T_{\text {in }}=(0.449 \pm 0.005) \mathrm{keV}$ (our Table 5) and $T_{\mathrm{in}}=(0.436 \pm 0.004) \mathrm{keV}$ (Table 2 in Cui et al. 1998); and $R_{\text {in }}=(2.54 \pm 0.06) r_{g}$ versus $(2.68 \pm 0.06) r_{g}$ (for $D=1.86 \mathrm{kpc}$, $M=14.8 M_{\odot}$, and $\left.i=27.1 ; r_{g} \equiv G M / c^{2}=21.9 \mathrm{~km}\right)$. For most of the other fit parameters, however, the differences between our results (Table 5) and theirs are quite significant: for example, for the width of the $\mathrm{Fe} \mathrm{K} \alpha$ line we find $\sigma=$ $(1.22 \pm 0.04) \mathrm{keV}$ versus $\sigma=(0.35 \pm 0.07) \mathrm{keV}$, and for the ionization parameter $\xi \approx 0$ versus $\xi>11911$. We attribute these differences to our use of response files that have been significantly updated and improved (Section 2). These new response files not only allowed us to extend the upper energy bound to $45 \mathrm{keV}$ (compared to the $30 \mathrm{keV}$ bound used by Cui et al.), but also greatly improved the fit: $\chi_{v}^{2}=0.88$ versus $\chi_{v}^{2}=1.47$. Furthermore, this comparison is understated because Cui et al. included 1\% systematic error in the PCU count rates whereas we used $0.5 \%$; for a systematic error of $1 \%$, our $\chi_{v}^{2}=0.68$.

Applying Model NR1 to SP2 and SP3 also gives very good fits (Table 5, Columns 5 and 6). However, comparing the results for all three spectra one finds a wide variation in the values of the disk temperature $T_{\text {in }}(0.27-0.54 \mathrm{keV})$ and inner disk radius $R_{\text {in }}\left(1.95-8.30 r_{g}\right)$. (We focus here on the parameters for the thermal component because this component ultimately delivers our key result, the spin of the black hole.) We find the performance of this model unsatisfactory for two principal reasons. First, it returns unlikely and near-zero values of the ionization parameter $\xi$ (which we have here fixed to zero); this is probably because both the reflection component and line feature are improperly modeled (see below). Second, this model implies an unrealistically strong line feature $(\mathrm{EW}=0.4-0.5 \mathrm{keV})$. Therefore, we now consider an improved model.

Model NR2. For the line feature, we replace the symmetric Gaussian profile with a skewed, relativistic profile via the LAOR model, while relativistically blurring the three other additive model components using KDBLUR (Table 4). The LAOR model and its companion convolution model KDBLUR have the serious limitation of assuming a fixed and extreme value of the spin parameter, $a_{*}=0.998$, and they therefore do not give a proper description of black holes with moderate or low spins. Nevertheless, we consider these models adequate for the present purposes because Cygnus X-1 is a rapidly spinning black hole and because the $\mathrm{Fe} \mathrm{K} \alpha$ line is only a cosmetic feature relative to the thermal continuum (Section 4). The merit of using LAOR/KDBLUR here is that the model is simple and the values of the line strength and ionization parameter returned by the fits are reasonable (Table 6). In the end, however, as in the case of the previous model, we find Model NR2 wanting because

\footnotetext{
27 As for our adopted model, we compute only the reflected component by restricting the fit parameter $s$ to negative values, which we here fix to -1 (see Section 3.2).
} 
Table 5

Fit Results for Model NR1 ${ }^{\mathrm{a}}$

\begin{tabular}{llcccc}
\hline \hline Number & Model & Parameter & SP1 & SP2 & SP3 \\
\hline 1 & DISKBB & $T_{\text {in }}$ & $0.449 \pm 0.005$ & $0.537 \pm 0.010$ & $0.270 \pm 0.013$ \\
2 & DISKBB & $R_{\text {in }}$ & $2.54 \pm 0.06$ & $1.95 \pm 0.10$ & $8.30 \pm 0.98$ \\
3 & CONST & $\cdots$ & $1.012 \pm 0.002$ & $1.071 \pm 0.013$ & $1.037 \pm 0.004$ \\
4 & TBABS & $N_{\mathrm{H}}$ & $0.675 \pm 0.008$ & $0.697 \pm 0.018$ & $0.862 \pm 0.022$ \\
5 & GAUSSIAN & $E_{\mathrm{L}}$ & $6.40 \pm 0.14$ & $6.40 \pm 0.14$ & $6.40 \pm 0.12$ \\
6 & GAUSSIAN & $\sigma$ & $1.22 \pm 0.04$ & $1.02 \pm 0.07$ & $1.02 \pm 0.04$ \\
7 & GAUSSIAN & $N_{\mathrm{L}}$ & $0.047 \pm 0.005$ & $0.078 \pm 0.011$ & $0.067 \pm 0.006$ \\
8 & GAUSSIAN & EW & 0.488 & 0.401 & 0.455 \\
9 & COMPBB & $T_{0}$ & $0.777 \pm 0.027$ & $0.947 \pm 0.049$ & $0.432 \pm 0.021$ \\
10 & COMPBB & $T_{\mathrm{e}}$ & $29.00 \pm 0.47$ & $21.33 \pm 1.24$ & $23.15 \pm 0.64$ \\
11 & COMPBB & $\tau$ & $1.08 \pm 0.02$ & $1.24 \pm 0.07$ & $0.80 \pm 0.05$ \\
12 & COMPBB & $N$ & $4887.9 \pm 741.6$ & $4074.1 \pm 978.0$ & $164572.0 \pm 48271.7$ \\
13 & PEXRIV & $\mathrm{X}_{\mathrm{Fe}}$ & $4.18 \pm 0.12$ & $2.08 \pm 0.25$ & $2.29 \pm 0.08$ \\
14 & PEXRIV & $N$ & $9.07 \pm 0.35$ & $13.17 \pm 0.84$ & $14.44 \pm 0.41$ \\
\hline 15 & & $\chi_{v}^{2}$ & $0.88(397 / 453)$ & $1.13(326 / 289)$ & $1.07(654 / 613)$ \\
\hline
\end{tabular}

Notes.

${ }^{\text {a }}$ For the model components given, the parameters from top to bottom are (1) inner disk temperature in keV; (2) inner disk radius in units of $r_{g} \equiv G M / c^{2}=21.9 \mathrm{~km}$ for $M=14.8 M_{\odot}$; (3) detector normalization constant relative to $R X T E$ PCU2; (4) hydrogen column density in units of $\mathrm{cm}^{-2}$; (5) central line energy in keV; (6) line width in keV; (7) line flux in units of photons $\mathrm{cm}^{-2} \mathrm{~s}^{-1}$; (8) equivalent width of line in $\mathrm{keV}$; (9) blackbody temperature in $\mathrm{keV}$; (10) electron temperature of corona in keV; (11) optical depth of corona; (12) normalization constant; (13) iron abundance relative to solar; (14) normalization constant; (15) reduced chi-square, total chi-square, and degrees of freedom, respectively. Details for the reflection component PEXRIV: apart from iron, the metal abundances are solar; the photon index is fixed at $\Gamma=2.5$, the value determined for Model NR3; the reflection scaling factor $s$ is fixed to -1 ; and the ionization parameter is fixed, $\xi=0$ (whereas, if fitted, $\xi \approx 0$ ).

$\mathrm{b}$ The line central energy is pegged in the fit.

Table 6

Fit Results for Model NR2 ${ }^{\mathrm{a}}$

\begin{tabular}{|c|c|c|c|c|c|}
\hline Number & Model & Parameter & SP1 & $\mathrm{SP} 2$ & SP3 \\
\hline 1 & DISKBB & $T_{\text {in }}$ & $0.509 \pm 0.003$ & $0.616 \pm 0.011$ & $0.446 \pm 0.002$ \\
\hline 2 & DISKBB & $R_{\text {in }}$ & $2.09 \pm 0.02$ & $1.61 \pm 0.05$ & $2.40 \pm 0.01$ \\
\hline 3 & CONST & $\ldots$ & $1.014 \pm 0.002$ & $1.083 \pm 0.011$ & $1.034 \pm 0.004$ \\
\hline 4 & TBABS & $N_{\mathrm{H}}$ & $0.701 \pm 0.007$ & $0.801 \pm 0.016$ & $0.717 \pm 0.006$ \\
\hline 5 & LAOR & $E_{\mathrm{L}}$ & $6.51 \pm 0.02$ & $6.49 \pm 0.04$ & $6.49 \pm 0.02$ \\
\hline 6 & LAOR & $q$ & $2.37 \pm 0.07$ & $2.54 \pm 0.11$ & $2.39 \pm 0.04$ \\
\hline 7 & LAOR & $N_{\mathrm{L}}$ & $0.012 \pm 0.001$ & $0.035 \pm 0.003$ & $0.032 \pm 0.001$ \\
\hline 8 & LAOR & EW & 0.125 & 0.176 & 0.219 \\
\hline 9 & СОМРВВ & $T_{0}$ & $1.060 \pm 0.015$ & $1.279 \pm 0.065$ & $0.522 \pm 0.001$ \\
\hline 10 & СОМРВВ & $T_{\mathrm{e}}$ & $33.64 \pm 0.64$ & $23.23 \pm 1.56$ & $27.20 \pm 0.42$ \\
\hline 11 & СОМРВВ & $\tau$ & $1.12 \pm 0.02$ & $1.22 \pm 0.08$ & $0.87 \pm 0.02$ \\
\hline 12 & СОМРВВ & $N$ & $1404.2 \pm 83.0$ & $1165.7 \pm 270.6$ & $59084.1 \pm 426.3$ \\
\hline 13 & PEXRIV & $\mathrm{X}_{\mathrm{Fe}}$ & $2.96 \pm 0.37$ & $2.31 \pm 0.38$ & $3.63 \pm 0.17$ \\
\hline 14 & PEXRIV & $\xi$ & $1168.0 \pm 115.4$ & $748.5 \pm 177.2$ & $1647.7 \pm 115.8$ \\
\hline 15 & PEXRIV & $N$ & $4.91 \pm 0.32$ & $14.51 \pm 0.98$ & $10.33 \pm 0.35$ \\
\hline 16 & & $\chi_{v}^{2}$ & $1.05(474 / 452)$ & $1.16(333 / 288)$ & $1.13(692 / 612)$ \\
\hline
\end{tabular}

Notes. ${ }^{a}$ Layout and parameter definitions very similar to Table 3, with two exceptions: (1) LAOR model substituted for GAUSSIAN and, correspondingly, the emissivity index $q$ is given in place of the line width $\sigma$; and (2) the ionization parameter $\xi$ is now a fit parameter rather than fixed to zero.

the parameters it returns for the crucial thermal component $\left(T_{\text {in }}\right.$ and $R_{\text {in }}$ ) differ quite significantly for the three spectra. We now discuss our most physical nonrelativistic model.

Model NR3. In addition to our criticisms of Models NR1 and NR2, we find these models structurally unsatisfactory because they incorporate two different and discrepant thermal temperatures: DISKBB's $T_{\text {in }} \sim 0.5 \mathrm{keV}$ and COMPTT's $T_{0} \sim$ $1.0 \mathrm{keV}$. In Model NR3, we solve this problem by replacing COMPBB with SIMPLR, which generates the Compton power-law component via a convolution by operating on an arbitrary source of seed photons, which in this case is the thermal component DISKBB prior to Compton scattering. Meanwhile, we retain the line model LAOR and the convolution model KDBLUR. Model NR3 is specified in Table 4, and our fit results are given in Table 7 . The fits are good and very comparable to those obtained using the other two models, even though Model NR3 uses one less fit parameter. We note that achieving these fits requires iron abundances that are $\approx 3.2-5.5$ times solar. The benefit of using this more self-consistent model is that it harmonizes the fitted values of the parameters of the thermal component: 
Table 7

Fit Results for Model NR3 ${ }^{\text {a }}$

\begin{tabular}{llcccc}
\hline \hline Number & Model & Parameter & SP1 & SP2 & SP3 \\
\hline 1 & DISKBB & $T_{\text {in }}$ & $0.532 \pm 0.001$ & $0.539 \pm 0.002$ & $0.543 \pm 0.001$ \\
2 & DISKBB & $R_{\text {in }}$ & $2.06 \pm 0.01$ & $2.30 \pm 0.01$ & $2.01 \pm 0.01$ \\
3 & CONST & $\ldots$ & $1.018 \pm 0.002$ & $1.078 \pm 0.010$ & $1.0295 \pm 0.0038$ \\
4 & TBABS & $N_{\mathrm{H}}$ & $0.680 \pm 0.004$ & $0.838 \pm 0.009$ & $0.699 \pm 0.004$ \\
5 & SIMPLR & $\Gamma$ & $2.206 \pm 0.011$ & $2.503 \pm 0.013$ & $2.486 \pm 0.005$ \\
6 & SIMPLR & $f_{\mathrm{SC}}$ & $0.176 \pm 0.002$ & $0.344 \pm 0.007$ & $0.317 \pm 0.003$ \\
7 & LAOR & $E_{\mathrm{L}}$ & $6.56 \pm 0.02$ & $6.44 \pm 0.04$ & $6.49 \pm 0.03$ \\
8 & LAOR & $q$ & $2.88 \pm 0.01$ & $2.36 \pm 0.06$ & $2.38 \pm 0.03$ \\
9 & LAOR & $N_{\mathrm{L}}$ & $0.018 \pm 0.001$ & $0.033 \pm 0.002$ & $0.030 \pm 0.001$ \\
10 & LAOR & EW & 0.189 & 0.164 & 0.208 \\
11 & PEXRIV & $\mathrm{X}_{\mathrm{Fe}}$ & $5.49 \pm 0.17$ & $3.24 \pm 0.13$ & $3.22 \pm 0.14$ \\
12 & PEXRIV & $\xi$ & $1147.3 \pm 100.4$ & $906.5 \pm 81.5$ & $1323.5 \pm 0.0$ \\
13 & PEXRIV & $N$ & $3.78 \pm 0.29$ & $15.38 \pm 1.17$ & $9.52 \pm 0.30$ \\
\hline 14 & & $\chi_{v}^{2}$ & $1.26(570 / 454)$ & $1.27(367 / 290)$ & $1.12(685 / 614)$ \\
\hline
\end{tabular}

Notes. ${ }^{a}$ For definitions of most of the parameters, see Tables 5 and 6 . The model components and parameters are the same as for Table 6 with one difference: the convolution model SIMPLR with two parameters has been substituted for the additive model сомРвв with four. The two parameters of SIMPLR are the photon power-law index $\Gamma$ and a normalization constant, which is the scattering fraction $f_{\mathrm{SC}}$.

Table 8

The Four Preliminary Relativistic Models

\begin{tabular}{|c|c|}
\hline Number & Model \\
\hline R1 & CRABCOR ${ }^{*} \mathrm{CONST}^{*}$ TBABS $($ KERRBB2+LAOR+KDBLUR $\otimes($ PEXRIV+COMPBB $))$ \\
\hline R2 & CRABCOR*CONST*TBABS(SIMPLR $\otimes$ KERRBB2+LAOR+KDBLUR $\otimes$ PEXRIV $)$ \\
\hline R3 & CRABCOR*CONST $*$ TBABS $($ KERRBB2+LAOR+KDBLUR $\otimes($ IREFLECT $\otimes$ COMPBB $))$ \\
\hline R4 & $\mathrm{CRABCOR}^{*} \mathrm{CONST}^{*} \mathrm{TBABS}(\mathrm{SIMPLR} \otimes \mathrm{KERRBB} 2+\mathrm{LAOR}+\mathrm{KDBLUR} \otimes($ IREFLECT $\otimes$ SIMPLC $))$ \\
\hline
\end{tabular}

Table 9

Fit Results for Model R1 ${ }^{\mathrm{a}}$

\begin{tabular}{llcccc}
\hline \hline Number & Model & Parameter & SP1 & SP2 & SP3 \\
\hline 1 & KERRBB 2 & $a_{*}$ & $0.9999_{-0.0001}^{+0}$ & $0.9999_{-0.0003}^{+0}$ & $0.9989_{-0.0021}^{+0.0003}$ \\
2 & KERRBB2 & $\dot{M}$ & $0.102 \pm 0.007$ & $0.143 \pm 0.030$ & $0.094 \pm 0.010$ \\
3 & const & $\ldots$ & $1.000 \pm 0.002$ & $1.004 \pm 0.014$ & $1.016 \pm 0.005$ \\
4 & TBABS & $N_{\mathrm{H}}$ & $0.760 \pm 0.008$ & $0.893 \pm 0.022$ & $0.759 \pm 0.010$ \\
5 & LAOR & $E_{\mathrm{L}}$ & $6.45 \pm 0.02$ & $6.42 \pm 0.04$ & $6.48 \pm 0.03$ \\
6 & LAOR & $q$ & $2.07 \pm 0.10$ & $2.02 \pm 0.20$ & $2.33 \pm 0.05$ \\
7 & LAOR & $N_{\mathrm{L}}$ & $0.011 \pm 0.001$ & $0.026 \pm 0.003$ & $0.030 \pm 0.001$ \\
8 & LAOR & EW & 0.106 & 0.131 & 0.208 \\
9 & COMPBB & $T_{0}$ & $1.023 \pm 0.005$ & $0.975 \pm 0.028$ & $0.559 \pm 0.002$ \\
10 & COMPBB & $T_{\mathrm{e}}$ & $24.23 \pm 0.47$ & $21.41 \pm 1.06$ & $24.23 \pm 0.57$ \\
11 & COMPBB & $\tau$ & $1.11 \pm 0.01$ & $1.32 \pm 0.07$ & $1.05 \pm 0.03$ \\
12 & COMPBB & $N$ & $1582.7 \pm 24.5$ & $3851.8 \pm 504.1$ & $39574.0 \pm 515.0$ \\
13 & PEXRIV & $\mathrm{X}_{\mathrm{Fe}}$ & $3.01 \pm 0.30$ & $2.87 \pm 0.38$ & $3.63 \pm 0.18$ \\
14 & PEXRIV & $\xi$ & $751.3 \pm 104.9$ & $652.1 \pm 169.2$ & $1226.3 \pm 0.1$ \\
15 & PEXRIV & $N$ & $4.87 \pm 0.31$ & $12.03 \pm 1.07$ & $10.87 \pm 0.40$ \\
\hline 16 & & $1.04(470 / 452)$ & $1.26(364 / 288)$ & $1.16(708 / 612)$ \\
\hline
\end{tabular}

Notes. ${ }^{\text {a }}$ For the model components given, the parameters from top to bottom are (1) spin parameter; (2) mass accretion rate in units of $10^{18} \mathrm{~g} \mathrm{~s}^{-1}$; (3) detector normalization constant relative to RXTE PCU2; (4) hydrogen column density in units of $\mathrm{cm}^{-2}$; (5) central line energy in $\mathrm{keV}$; (6) emissivity index; (7) line flux in units of photons $\mathrm{cm}^{-2} \mathrm{~s}^{-1}$; (8) equivalent width of line in keV; (9) blackbody temperature in keV; (10) electron temperature of corona in keV; (11) optical depth of corona; (12) normalization constant; (13) iron abundance relative to solar; (14) ionization parameter; (15) normalization constant; (16) reduced chi-square, total chi-square, and degrees of freedom, respectively. Details for the reflection component PEXRIV: apart from iron, the metal abundances are solar, and the photon index is fixed at $\Gamma=2.5$, which is the value determined for our adopted model (Table 2). 
Table 10

Fit Results for Model R2 ${ }^{\mathrm{a}}$

\begin{tabular}{llcccc}
\hline \hline Number & Mode1 & Parameter & SP1 & SP2 & SP3 \\
\hline 1 & KERRBB2 & $a_{*}$ & $0.9989_{-0.0003}^{+0.003}$ & $0.9998_{-0.0036}^{+0}$ & $0.9999_{-0.0001}^{+0}$ \\
2 & KERRBB2 & $\dot{M}$ & $0.116 \pm 0.003$ & $0.146 \pm 0.025$ & $0.124 \pm 0.010$ \\
3 & const & $\ldots$ & $1.006 \pm 0.002$ & $1.042 \pm 0.013$ & $0.972 \pm 0.004$ \\
4 & TBABS & $N_{\mathrm{H}}$ & $0.758 \pm 0.006$ & $0.841 \pm 0.018$ & $0.764 \pm 0.010$ \\
5 & SIMPLR & $\Gamma$ & $2.295 \pm 0.011$ & $2.507 \pm 0.012$ & $2.525 \pm 0.008$ \\
6 & SIMPLR & $f_{\mathrm{SC}}$ & $0.179 \pm 0.002$ & $0.328 \pm 0.009$ & $0.299 \pm 0.003$ \\
7 & LAOR & $E_{\mathrm{L}}$ & $6.54 \pm 0.03$ & $6.41 \pm 0.04$ & $6.51 \pm 0.03$ \\
8 & LAOR & $q$ & $2.87 \pm 0.02$ & $2.27 \pm 0.07$ & $2.41 \pm 0.05$ \\
9 & LAOR & $N_{\mathrm{L}}$ & $0.014 \pm 0.001$ & $0.032 \pm 0.002$ & $0.028 \pm 0.001$ \\
10 & LAOR & EW & 0.148 & 0.154 & 0.187 \\
11 & PEXRIV & $\mathrm{X}_{\mathrm{Fe}}$ & $6.23 \pm 0.17$ & $3.06 \pm 0.12$ & $3.60 \pm 0.15$ \\
12 & PEXRIV & $\xi$ & $726.4 \pm 39.5$ & $981.7 \pm 103.4$ & $1163.5 \pm 73.4$ \\
13 & PEXRIV & $N$ & $6.91 \pm 0.44$ & $15.23 \pm 1.01$ & $12.28 \pm 0.53$ \\
\hline 14 & & $\chi_{v}^{2}$ & $1.24(561 / 454)$ & $1.28(371 / 290)$ & $1.18(725 / 614)$ \\
\hline
\end{tabular}

Notes. ${ }^{a}$ For definitions of most of the parameters, see Table 9. There are two distinctions between this table and Table 9: (1) the convolution model SIMPLR with two parameters has been substituted for the additive model COMPBB with four. The two parameters of SIMPLR are the photon power-law index $\Gamma$ and a normalization constant, which is the scattering fraction $f_{\mathrm{SC}}$. (2) The photon index in PEXRIV is not fixed, rather it is linked to the photon index in SIMPLR.

Table 11

Fit Results for Model R3 ${ }^{\mathrm{a}}$

\begin{tabular}{llcccc}
\hline \hline Number & Model & Parameter & SP1 & SP2 & SP3 \\
\hline 1 & KERRBB2 & $a_{*}$ & $0.9989_{-0.0007}^{+0.003}$ & $0.9999_{-0.0003}^{+0}$ & $0.9888_{-0.0066}^{+0.0037}$ \\
2 & KERRBB2 & $\dot{M}$ & $0.110 \pm 0.004$ & $0.145 \pm 0.031$ & $0.098 \pm 0.008$ \\
3 & const & $\ldots$ & $1.001 \pm 0.002$ & $0.984 \pm 0.015$ & $1.026 \pm 0.004$ \\
4 & TBABS & $N_{\mathrm{H}}$ & $0.733 \pm 0.006$ & $0.820 \pm 0.025$ & $0.670 \pm 0.011$ \\
5 & LAOR & $E_{\mathrm{L}}$ & $6.43 \pm 0.02$ & $6.42 \pm 0.04$ & $6.45 \pm 0.03$ \\
6 & LAOR & $q$ & $1.78 \pm 0.14$ & $1.83 \pm 0.28$ & $2.26 \pm 0.06$ \\
7 & LAOR & $N_{\mathrm{L}}$ & $0.011 \pm 0.001$ & $0.024 \pm 0.003$ & $0.030 \pm 0.001$ \\
8 & LAOR & EW & 0.109 & 0.117 & 0.204 \\
9 & COMPBB & $T_{0}$ & $0.942 \pm 0.010$ & $1.004 \pm 0.017$ & $0.518 \pm 0.001$ \\
10 & COMPBB & $T_{\mathrm{e}}$ & $26.25 \pm 0.43$ & $22.85 \pm 0.61$ & $26.25 \pm 0.48$ \\
11 & COMPBB & $\tau$ & $1.19 \pm 0.02$ & $1.24 \pm 0.04$ & $0.90 \pm 0.03$ \\
12 & COMPBB & $N$ & $2212.8 \pm 99.1$ & $3301.5 \pm 234.5$ & $59300.0 \pm 532.3$ \\
13 & IREFLECT & $\mathrm{X}_{\mathrm{Fe}}$ & $2.20 \pm 0.19$ & $2.02 \pm 0.26$ & $3.51 \pm 0.18$ \\
14 & IREFLEC & $s$ & $0.36 \pm 0.02$ & $0.72 \pm 0.03$ & $0.72 \pm 0.03$ \\
15 & IREFLECT & $\xi$ & $84.2 \pm 9.0$ & $59.2 \pm 12.5$ & $65.5 \pm 6.8$ \\
\hline 16 & & $\chi_{v}^{2}$ & $1.07(483 / 452)$ & $1.25(358 / 288)$ & $1.14(695 / 612)$ \\
\hline
\end{tabular}

Notes.

${ }^{a}$ Here, we have substituted the convolution model IREFLECT for the additive reflection model PEXRIV. These models have two parameters in common, the iron abundance and ionization parameter, and one that differs, namely, the normalization of PEXRIV is replaced by the reflection scaling factor $s$. For the definitions of all other parameters, see Tables 9 and 10 .

${ }^{\mathrm{b}}$ In applying IREFLECT, the reflection scaling factor $s$ is negative; here we give absolute values of $s$.

$T_{\text {in }}=0.532,0.539$, and $0.543 \mathrm{keV}$ and $R_{\text {in }}=2.06,2.30$, and $2.01 r_{g}$ for SP1, SP2, and SP3, respectively. Meanwhile, the smallness of the inner disk radius $R_{\text {in }}$ is suggestive of the high spin revealed by our relativistic analysis (see Section 3).

\section{APPENDIX B}

\section{RELATIVISTIC ANALYSIS}

Four preliminary Models RI-R4. We now consider a progression of four models that are all built around our relativistic disk model KERRBB2 (Section 3.2). These models progress toward our adopted model (Section 3.2) in the sense that Model $\mathrm{R} 1$ is the most primitive and our adopted model is the most physically realistic. All four of these models and our adopted model give very similar results for the parameter of interest, namely, $a_{*}$, indicating that our key result, the extreme spin of Cygnus X-1, is quite insensitive to the details of the analysis.

The four preliminary models R1-R4 are defined in Table 8 . Every individual component in all four models has already 
Table 12

Fit Results for Model R4 ${ }^{\mathrm{a}}$

\begin{tabular}{llcccc}
\hline \hline Number & Model & Parameter & SP1 & SP2 & SP3 \\
\hline 1 & KERRBB2 & $a_{*}$ & $0.9987_{-0.0005}^{+0.0004}$ & $0.9997_{-0.0026}^{+0.0001}$ & $0.9999_{-0.0001}^{+0}$ \\
2 & KERRBB2 & $\dot{M}$ & $0.115 \pm 0.004$ & $0.143 \pm 0.026$ & $0.122 \pm 0.011$ \\
3 & Const & $\ldots$ & $1.000 \pm 0.002$ & $1.037 \pm 0.013$ & $0.726 \pm 0.003$ \\
4 & TBABS & $N_{\mathrm{H}}$ & $0.704 \pm 0.006$ & $0.761 \pm 0.022$ & $0.687 \pm 0.010$ \\
5 & SIMPLR & $\Gamma$ & $2.284 \pm 0.010$ & $2.525 \pm 0.012$ & $2.551 \pm 0.011$ \\
6 & SIMPLR & $f_{\mathrm{SC}}$ & $0.227 \pm 0.006$ & $0.329 \pm 0.013$ & $0.308 \pm 0.006$ \\
7 & LAOR & $E_{\mathrm{L}}$ & $6.54 \pm 0.03$ & $6.42 \pm 0.04$ & $6.48 \pm 0.04$ \\
8 & LAOR & $q$ & $2.85 \pm 0.02$ & $2.26 \pm 0.07$ & $2.33 \pm 0.07$ \\
9 & LAOR & $N_{\mathrm{L}}$ & $0.016 \pm 0.001$ & $0.030 \pm 0.002$ & $0.025 \pm 0.002$ \\
10 & LAOR & EW & 0.161 & 0.150 & 0.165 \\
11 & IREFLECT & $\mathrm{X}_{\mathrm{Fe}}$ & $5.33 \pm 0.15$ & $3.16 \pm 0.12$ & $3.63 \pm 0.14$ \\
12 & IREFLECT & $s$ & $1.00 \pm 0.04$ & $0.89 \pm 0.03$ & $0.95 \pm 0.03$ \\
13 & IREFLECT & $\xi$ & $148.0 \pm 14.7$ & $53.6 \pm 7.9$ & $60.8 \pm 8.2$ \\
\hline 14 & & $\chi_{v}^{2}$ & $1.20(545 / 454)$ & $1.28(370 / 290)$ & $1.17(720 / 614)$ \\
\hline
\end{tabular}

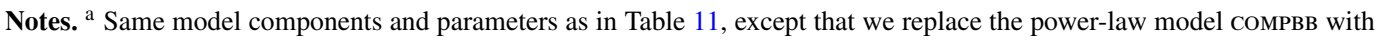
the convolution model SIMPLR, thereby reducing the four parameters of the former model component to two, namely, the photon power-law index $\Gamma$ and the normalization constant $f_{\mathrm{SC}}$.

been described either in Section 3.2 or in Appendix A. There are only two combinations of model components that are new and that have not been used elsewhere, namely, the reflection components in Models R3 and R4. Their core power-law components are, respectively, COMPBB and SIMPLC, each of which is convolved in turn with IREFLECT and KDBLUR.

The results for Models R1-R4 are presented, respectively, in Tables 9-12. Comparing the results for Models R1 and R2, which both employ the widely used reflection model PEXRIV, Model R2 is preferred because it gives very comparable values of reduced chi-square using two fewer fit parameters, and its reflection component SIMPLR self-consistently generates the power law with no need for the second thermal component required by СОмРвв. Furthermore, in the case of the nonrelativistic analysis (Appendix A), it was shown that SIMPLR delivers consistent values of $R_{\text {in }}$ and $T_{\text {in }}$, which COMPBB fails to do.

Tables 11 and 12 summarize our results for Models R3 and $\mathrm{R} 4$, which use the convolution reflection model IREFLECT in place of the additive model PEXRIV. While both reflection models are based on the same physics, the virtue of IREFLECT is that it can take any shape of spectrum as input, whereas PEXRIV requires a power-law input spectrum. In considering Models R1-R4, we find Model R4 to be superior because it fits the data as well as the other models while using the fewest parameters, and it is the most self-consistent (see Section 3.2).

In a final step, as described in Section 3.2, we arrive at our adopted model by replacing the flawed model components LAOR and KDBLUR (see Appendix A) by KERRDISK and KERRCONV. The virtue of KERRDISK/KERRCONV compared to LAOR/KDBLUR is that the spin parameter is free, allowing the GR metric to be calculated properly. We use a single, linked value of $a_{*}$ in fitting KERRDISK/KERRCONV and KERRBB2. An inspection of Tables 9- 12 and Table 3 shows that the key quantity, namely, the spin parameter, is precisely determined and near unity for Models R1-R4 and our adopted model. This indicates that the details of how one models the power-law and reflected components has little affect on the determination of $a_{*}$.

\section{REFERENCES}

Arnaud, K. A. 1996, in ASP Conf. Ser. 101, Astronomical Data Analysis Software and Systems V, ed. G. H. Jacoby \& J. Barnes (San Francisco, CA: ASP), 17

Axelsson, M., Borgonovo, L., \& Larsson, S. 2005, A\&A, 438, 999

Axelsson, M., Church, R. P., Davies, M. B., Levan, A. J., \& Ryde, F. 2011, MNRAS, 412, 2260

Bałucińska-Church, M., Church, M. J., Charles, P. A., et al. 2000, MNRAS, 311, 861

Bardeen, J. M. 1970, Nature, 226, 64

Bardeen, J. M., Press, W. H., \& Teukolsky, S. A. 1972, ApJ, 178, 347

Blandford, R. D., \& Znajek, R. L. 1977, MNRAS, 179, 433

Blum, J. L., Miller, J. M., Fabian, A. C., et al. 2009, ApJ, 706, 60

Bolton, C. T. 1972, Nature, 240, 124

Bowyer, S., Byram, E. T., Chubb, T. A., \& Friedman, H. 1965, Science, 147, 394

Brenneman, L. W., \& Reynolds, C. S. 2006, ApJ, 652, 1028

Brocksopp, C., Tarasov, A. E., Lyuty, V. M., \& Roche, P. 1999, A\&A, 343, 861

Canizares, C. R., Davis, J. E., Dewey, D., et al. 2005, PASP, 117, 1144

Christodoulou, D., \& Ruffini, R. 1971, Phys. Rev. D, 4, 3552

Cui, W., Ebisawa, K., Dotani, T., \& Kubota, A. 1998, ApJ, 493, L75

Dauser, T., Wilms, J., Reynolds, C. S., \& Brenneman, L. W. 2010, MNRAS, 409, 1534

Davis, S. W., Blaes, O. M., Hubeny, I., \& Turner, N. J. 2005, ApJ, 621, 372

Davis, S. W., \& Hubeny, I. 2006, ApJS, 164, 530

Done, C., Gierliński, M., \& Kubota, A. 2007, A\&AR, 15, 1

Dotani, T., Inoue, H., Mitsuda, K., et al. 1997, ApJ, 485, L87

Dubus, G., Hameury, J.-M., \& Lasota, J.-P. 2001, A\&A, 373, 251

Duro, R., Dauser, T., Wilms, J., et al. 2011, A\&A, 533, L3

Fabian, A. C., Rees, M. J., Stella, L., \& White, N. E. 1989, MNRAS, 238, 729

Fender, R., \& Belloni, T. 2004, ARA\&A, 42, 317

Fragos, T., Tremmel, M., Rantsiou, E., \& Belczynski, K. 2010, ApJ, 719, L79

Garmire, G. P., Bautz, M. W., Ford, P. G., Nousek, J. A., \& Ricker, G. R., Jr. 2003, Proc. SPIE, 4851, 28

Gierliński, M., Zdziarski, A. A., Poutanen, J., et al. 1999, MNRAS, 309, 496

Gou, L., McClintock, J. E., Liu, J., et al. 2009, ApJ, 701, 1076

Gou, L., McClintock, J. E., Steiner, J. F., et al. 2010, ApJ, 718, L122

Hanke, M., Wilms, J., Nowak, M. A., Barragán, L., \& Schulz, N. S. 2010, A\&A, $509, \mathrm{~L} 8$

Hanke, M., Wilms, J., Nowak, M. A., et al. 2009, ApJ, 690, 330

Homan, J., \& Belloni, T. 2005, Ap\&SS, 300, 107

King, A. R., \& Kolb, U. 1999, MNRAS, 305, 654

Kulkarni, A. K., Penna, R. F., Shcherbakov, R. V., et al. 2011, MNRAS, 414, 1183

Laor, A. 1991, ApJ, 376, 90

Larsson, J., Miniutti, G., Fabian, A. C., et al. 2008, MNRAS, 384, 1316 
Li, L., Narayan, R., \& McClintock, J. E. 2009, ApJ, 691, 847

Li, L., Zimmerman, E. R., Narayan, R., \& McClintock, J. E. 2005, ApJS, 157, 335

Liu, J., McClintock, J. E., Narayan, R., Davis, S. W., \& Orosz, J. A. 2008, ApJ, 679, L37 (erratum 2010, ApJ, 719, L109)

Liu, J., McClintock, J. E., Narayan, R., Davis, S. W., \& Orosz, J. A. 2010, ApJ, 719, L109

Magdziarz, P., \& Zdziarski, A. A. 1995, MNRAS, 273, 837

Makishima, K., Maejima, Y., Mitsuda, K., et al. 1986, ApJ, 308, 635

Makishima, K., Tashiro, M., Ebisawa, K., et al. 1996, PASJ, 48, 171

McClintock, J. E., Narayan, R., Davis, S. W., et al. 2011, Class. Quantum Grav., 28, 114009

McClintock, J. E., Shafee, R., Narayan, R., et al. 2006, ApJ, 652, 518

Miller, J. M., Fabian, A. C., Nowak, M. A., \& Lewin, W. H. G. 2005, in Proc. 10th Marcel Grossmann Meeting on General Relativity, ed. M. Novello \& P. Bergliaffa (Hackensack, NJ: World Scientific), 1296

Miller, J. M., Reynolds, C. S., Fabian, A. C., Miniutti, G., \& Gallo, L. C. 2009, ApJ, 697, 900

Mirabel, I. F., \& Rodrigues, I. 2003, Science, 300, 1119

Mirabel, I. F., \& Rodríguez, L. F. 1994, Nature, 371, 46

Mitsuda, K., Inoue, H., Koyama, K., et al. 1984, PASJ, 36, 741

Moreno Méndez, E. 2011, MNRAS, 413, 183

Nishimura, J., Mitsuda, K., \& Itoh, M. 1986, PASJ, 38, 819

Noble, S. C., Krolik, J. H., \& Hawley, J. F. 2010, ApJ, 711, 959

Novikov, I. D., \& Thorne, K. S. 1973, in Black Holes, Gordan, and Breach, ed. C. De Witt \& B. De Witt (New York: Gordon \& Breach), 343

Orosz, J. A., McClintock, J. E., Aufdenberg, J. P., et al. 2011, ApJ, 742, 84

Penna, R. F., McKinney, J. C., Narayan, R., et al. 2010, MNRAS, 408, 752
Penrose, R. 1969, Nuovo Cimento Riv. Ser., 1, 252

Reid, M. J., McClintock, J. E., Narayan, R., et al. 2011, ApJ, 742, 83

Remillard, R. A., \& McClintock, J. E. 2006, ARA\&A, 44, 49

Reynolds, C. S., \& Fabian, A. C. 2008, ApJ, 675, 1048

Reynolds, C. S., \& Nowak, M. A. 2003, Phys. Rep., 377, 389

Riffert, H., \& Herold, H. 1995, ApJ, 450, 508

Romano, P., Cusumano, G., Campana, S., et al. 2005, Proc. SPIE, 5898, 369

Ross, R. R., \& Fabian, A. C. 2005, MNRAS, 358, 211

Shafee, R., McClintock, J. E., Narayan, R., et al. 2006, ApJ, 636, L113

Shafee, R., McKinney, J. C., Narayan, R., et al. 2008, ApJ, 687, L25

Smak, J. 1999, Acta Astron., 49, 391

Smak, J. I. 1998, Acta Astron., 48, 677

Smith, R. K., Edgar, R. J., \& Shafer, R. A. 2002, ApJ, 581, 562

Steiner, J. F., McClintock, J. E., Remillard, R. A., Narayan, R., \& Gou, L. 2009a, ApJ, 701, L83

Steiner, J. F., McClintock, J. E., Remillard, R. A., et al. 2010, ApJ, 718, L117

Steiner, J. F., Narayan, R., McClintock, J. E., \& Ebisawa, K. 2009b, PASP, 121, 1279

Steiner, J. F., et al. 2011, MNRAS, 416, 941

Stella, L., Vietri, M., \& Morsink, S. M. 1999, ApJ, 524, L63

Toor, A., \& Seward, F. D. 1974, AJ, 79, 995

Webster, B. L., \& Murdin, P. 1972, Nature, 235, 37

Wilms, J., Allen, A., \& McCray, R. 2000, ApJ, 542, 914

Wong, T.-W., Valsecchi, F., Fragos, T., \& Kalogera, V. 2011, ApJ, submitted (arXiv:1107.5585v1)

Zhang, S. N., Cui, W., \& Chen, W. 1997, ApJ, 482, L155

Zimmerman, E. R., Narayan, R., McClintock, J. E., \& Miller, J. M. 2005, ApJ, 618, 832 